Revista Brasileira de Cartografia

ISSN 1808-0936 | https://doi.org/10.14393/revbrascartogr

Sociedade Brasileira de Cartografia, Geodésia, Fotogrametria e Sensoriamento Remoto

\title{
Cartas Náuticas com Modelos SEP: Evolução Histórica, e Perspectivas para Hidrografia Brasileira
}

\section{Nautical Charts with SEP Models: Historic Evolution and Perspectives for the Brazilian Hydrography}

Felipe Rodrigues Santana ${ }^{1}$, Claudia Pereira Krueger ${ }^{2}$, Tulio Alves Santana ${ }^{3}$, Guilherme Antonio Gomes Nascimento ${ }^{4}$ e Aluízio Maciel Oliveira Junior ${ }^{5}$

1 Universidade Federal do Paraná, Programa de Pós-Graduação Ciências Geodésicas, Curitiba, Brasil. felipesantana33@gmail.com. ORCID: https://orcid.org/0000-0001-6410-7999

2 Universidade Federal do Paraná, Programa de Pós-Graduação Ciências Geodésicas Curitiba, Brasil. cpkrueger64@gmail.com. ORCID: http://orcid.org/0000-0002-4839-1317

3 Universidade Federal de Uberlândia/ Universidade Federal do Paraná, Monte Carmelo, Brasil. tulio.santana@ufu.br. ORCID: https://orcid.org/0000-0002-4429-9409

4 Marinha do Brasil, Centro de Hidrografia da Marinha, Niterói, Brasil. guilhermenascimento@ hotmail.com. ORCID: https://orcid.org/0000-0003-0579-2070

5 Delfos Assessoria e Serviços Marítimos, Diretoria Técnica, Niteroí, Brasil. aluizio94@outlook.com. ORCID: https://orcid.org/0000-0003-3625-5809

Recebido: 08.2020 | Aceito: 11.2020

Resumo: Os avanços das técnicas GNSS de alta precisão permitiram a diminuição das incertezas verticais dos levantamentos batimétricos, com uma melhor determinação do heave, do calado dinâmico e redução dos erros cotidais. Contudo, é necessário a determinação de um modelo de separação (SEP) entre o Datum da Carta Náutica (DCN) e o elipsoide de referência. Neste artigo será apresentada uma evolução histórica sobre o desenvolvimento de modelos SEP no mundo através de sete países: Estados Unidos, Canadá, Holanda, Arábia Saudita, Colômbia, Inglaterra e Brasil. O resultado das estratégias adotadas por países estrangeiros mostra incertezas de modelos SEP variando de 6,6 cm a 22,6 cm em relação ao ITRF. No caso do Brasil, é descrito um estudo pioneiro para o SEP da Baía de Guanabara, onde foi encontrada uma diferença média de $2,5 \mathrm{~cm}$ com um desvio padrão de $5,1 \mathrm{~cm}$ entre a superfície gerada com método de redução tradicional e por maré-GPS. Para uma cobertura nacional, é apresentado o projeto Alt-Bat, que prevê a utilização do geoide como referência vertical para os modelos hidrodinâmicos. Quanto às perspectivas, percebe-se um ciclo virtuoso para o desenvolvimento portuário: o investimento em dados ambientais, fornece uma maior acurácia de modelos SEP e menor incerteza dos levantamentos, propiciando maior calado, possibilidade do aumento no fluxo de cargas em portos e mais recursos para investimento. Por fim são apresentados os desafios a serem superados para a Hidrografia brasileira visando a determinação do DCN com uma acurácia de $10 \mathrm{~cm}$ em relação ao ITRF. Os modelos SEP são fundamentais para a integração de informações provenientes de diferentes referenciais verticais proporcionando uma navegação segura e gestão de processos costeiros.

Palavras-chave: Hidrografia. GNSS. Modelagem. Maré-GPS. Altimetria Satelital.

Abstract: Advances in high-precision GNSS techniques have allowed the reduction of vertical uncertainties in bathymetric surveys, with a better determination of the heave, the dynamic draft and the reduction of co-tidal errors. However, it is necessary to determine a separation model (SEP) between the Chart Datum and the reference ellipsoid. This article will present a historical evolution on the development of SEP models in the world across seven countries: the United States, Canada, Netherlands, Saudi Arabia, Colombia, England and Brazil. Research from foreign countries shows uncertainties in SEP models ranging from $6.6 \mathrm{~cm}$ to $22.6 \mathrm{~cm}$ in relation to the ITRF. In the case of Brazil, a pioneering study for the SEP of Guanabara Bay is described, where an average difference of $2.5 \mathrm{~cm}$ was found with a standard deviation of $5.1 \mathrm{~cm}$ between the surface generated with the traditional reduction method and by GPS-tide. For national coverage, the Alt-Bat project is presented, which provides the use of the geoid as a vertical reference for hydrodynamic models. As for the prospects, there is a virtuous cycle for port development: investment in environmental data, provides greater accuracy of SEP models and less uncertainty of surveys, providing greater draft, possibility of increased cargo flow in ports and more resources for investment. SEP models are fundamental for the gathering of information from different vertical references, providing safe navigation and management of coastal process.

Keywords: Hydrography. GNSS. Modeling. GPS-Tide. Satellite Altimetry. 


\section{INTRODUÇÃ̃O}

Em 2019 Organização das Nações Unidas (ONU) considerou o período de 2021 a 2030 como a "Década dos Oceanos" para o desenvolvimento sustentável, tendo entre seus objetivos um oceano seguro, previsível, transparente e produtivo, onde os dados marinhos pudessem ser mapeados, previstos, divulgados e seus recursos explorados sustentavelmente. Neste contexto global da Economia Azul, com intuito de aumentar a segurança da navegação e reduzir a probabilidade de acidentes náuticos, a Organização Hidrográfica Internacional (OHI) passou a incluir a Ordem Exclusiva nas Especificações para Levantamentos Hidrográficos (S-44 $6^{\mathrm{a}}$ edição), com parâmetros de incertezas verticais mais rigorosos onde a Folga Abaixo da Quilha (FAQ) da embarcação é crítica. (IOC/UNESCO et al., 2011; UNESCO, 2019; OHI; 2020a).

Alguns países já passaram a adotar tais critérios em suas especificações (Tabela 1). No Brasil, a NORMAM-25 interioriza os padrões internacionais, todavia inclui até a Ordem Especial (DHN, 2017).

Tabela 1 - Padrões mínimos de incerteza para LH a 10m de profundidade, a um nível de confiança de 95\%.

\begin{tabular}{c|c|c|c|c|c}
\hline Critério $(\mathbf{m})$ & Ordem 2 & Ordem 1b & Ordem 1a & Ordem Especial & $\begin{array}{c}\text { Ordem } \\
\text { Exclusiva }\end{array}$ \\
\hline Incerteza Vertical Total & 1,03 & 0,52 & 0,52 & 0,26 & 0,17 \\
Incerteza Horizontal Total & 21,00 & 5,25 & 5,25 & 2,00 & 1,00 \\
Linha de costa & 10 & 10 & 10 & 10 & 5 \\
\hline
\end{tabular}

Fonte: DHN (2017) e CHS (2013).

A redução das incertezas dos levantamentos se reveste de importância com o advento do Sistema de Informação e Exibição de Cartas Eletrônicas (ECDIS - Eletronic Chart Display and Information System) e das cartas náuticas da classe S-10X, em que informações como tipo de fundo, maré, vento, corrente e ondas podem ser integradas em tempo real e a profundidade ser visualizada de maneira dinâmica. Tais dados ambientais, utilizados em conjunto com as técnicas do Sistema Global de Navegação por Satélites (GNSS - Global Navigation Satellite System) de alta precisão, como GPS-RTK (OTF - On The Fly), permitem as embarcações navegarem de acordo com a FAQ de forma segura (Figura 1), avaliando continuamente o risco de contato do navio com o fundo, aumentando sua capacidade de carga, reduzindo custos da viagem e a poluição ao meio ambiente, o que já é uma realidade em portos como Rotterdam, na Holanda, e Santos no Brasil. Entretanto, torna-se premente estabelecer o grau de incerteza dos sensores de monitoramento ambiental utilizados e dos dados batimétricos disponíveis, bem como a frequência dos levantamentos (WELLS; KLEUSBERG; VANÍČEK, 1996; KRUEGER, 1996; DPC, 2019; CONAPRA, 2020).

Figura 1 - A margem de segurança devido ao tipo de fundo é obtida de forma confiável, a partir do conhecimento da medição da profundidade do mar, do nível zero da referência da profundidade medida, da previsão da maré e da posição vertical do navio.

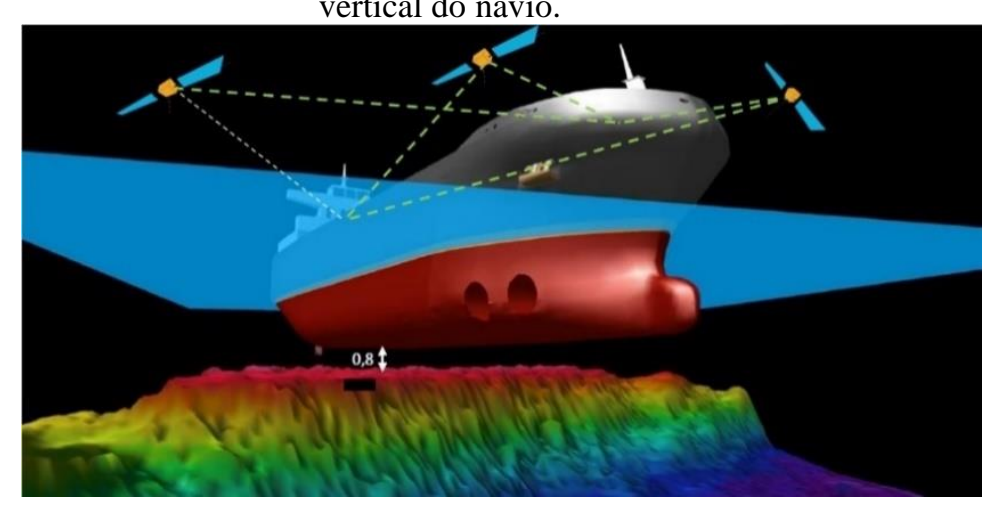

Fonte: FAMOS (2020)

O uso de receptores GNSS de alta precisão permite reduzir as incertezas da batimetria, haja visto que as correções de heave, calado dinâmico e marés podem ser feitas a partir da variação das altitudes elipsoidais da embarcação (Figura 2), o que reduz os erros cotidais. Estes erros consistem na diferença entre a variação da maré medida na estação maregráfica e no local onde se realiza a sondagem. Dessa forma, também os esforços logísticos dos levantamentos hidrográficos são reduzidos consideravelmente, uma vez que dispensa a 
necessidade de manutenção de estações maregráficas na costa. Com isto, os levantamentos se tornam mais rápidos, baratos e com menor incerteza nos dados. (ZIEBART et al. 2007; RAMOS, 2007; FIG, 2014).

Contudo, para efetuar a redução de sondagem, necessária em levantamentos hidrográficos, por meio de maré-GPS, é necessário o desenvolvimento de um modelo de separação (SEP) entre o Datum da Carta Náutica $(D C N)^{1}$ e o elipsoide de referência. Este elipsoide deve ser orientado e fixado para uma época particular em relação à Rede de Referência Terrestre Internacional (ITRF - International Terrestrial Reference Frame) (FIG, 2006).

Figura 2 - Esquema de componentes verticais envolvidas no levantamento hidrográfico.

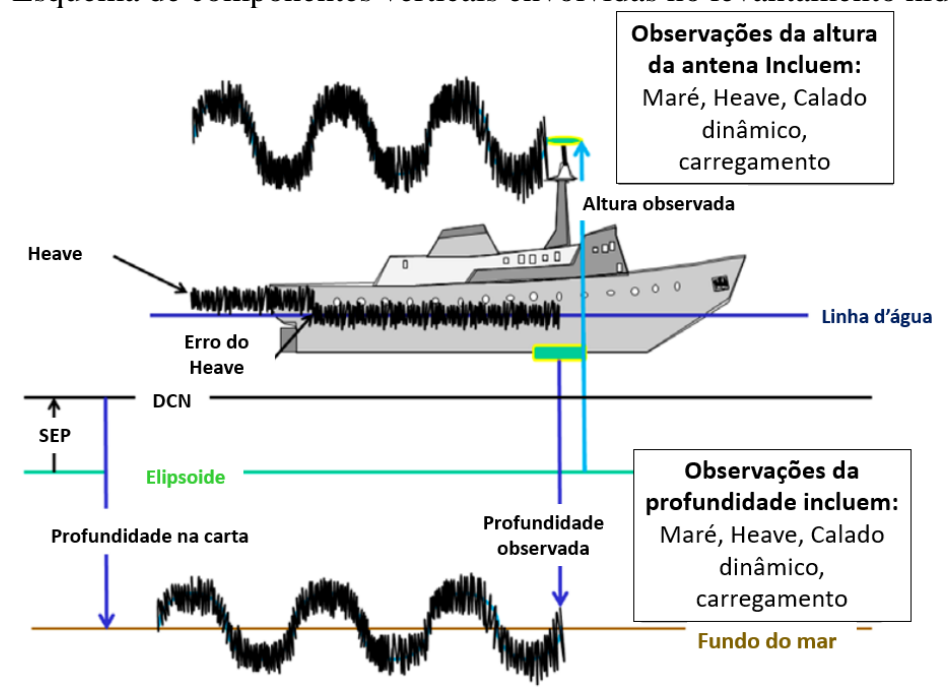

Fonte: Adaptada de FIG (2014).

Além da hidrografia, o SEP também é útil para a integração dos referenciais verticais terrestres e marítimos. No Brasil, por exemplo, os dados de batimetria são referenciados ao Nível de Redução (NR) da carta náutica, correspondendo às médias das baixa-mares de sizígia; os dados de linha de costa coletados em relação às médias da preamar das marés de sizígia (DHN, 2017); os de topografia, aos Níveis Médios do Mar (NMMs), observados em Imbituba-SC e em Santana-AP, e materializados por todo país pela Rede Vertical de Referência do Brasil (RVRB) do Sistema Geodésico Brasileiro (SGB) (LUZ, 2016). Todos são referenciais de estações maregráficas, determinados em um ponto e período específico, variando de local para local, dificultando a integração dos dados em vastas áreas. Já a utilização do SEP na zona costeira permite a integração dos dados a um referencial único e estável, como o Sistema Geodésico de Referência de 1980 (GRS80 - Geodetic Reference System 1980), orientado e fixado a uma determinada época do ITRF (FIG, 2006).

A partir da definição de um SEP é possível estimar modelos digitais de elevação mais precisos, que servem para vários fins, como restauração de habitats, mapeamento de corais e do fundo marinho, estudos de erosão, construção civil, mensuração da elevação do nível do mar, modelagem do impacto de ondas e tempestades na costa, planos de enchentes e de evacuação. Cita-se ainda a mensuração da alteração da linha costa e delimitação da fronteira marinha. Em regiões como Maricá-RJ, por exemplo, onde foi medida uma variação de 1,7 m em um ano, a acurácia de tal modelo torna-se essencial (PARKER, 2002; OSILIERI; SEOANE; DIAS, 2020). Quanto aos levantamentos hidrográficos, a redução das incertezas potencializa um círculo virtuoso para a economia do país, pois quanto mais segura for a navegação, maior o potencial de movimentação de cargas em um porto, o que pode contribuir ainda, com a modernização das infraestruturas portuárias. Neste cenário, os modelos SEP são essenciais para o desenvolvimento da economia azul de uma nação. Contudo, é necessário o investimento em uma infraestrutura que possibilite a obtenção de dados maregráficos, geodésicos e gravimétricos consistentes. (MMA, 2018; SUPRG, 2018).

Para o aprofundamento destas temáticas será apresentada na seção 2 uma revisão sobre as superfícies de referências verticais necessárias para a definição do SEP. A seção 3, por sua vez, apresentará uma evolução histórico-científica ao longo de duas décadas com um panorama de modelos SEP desenvolvidos no mundo.

${ }^{1}$ Em língua inglesa, o DCN é amplamente conhecido como Chart Datum (CD). 
Internacionalmente, serão explicadas as estratégias internacionais empregadas por alguns países para o cálculo das incertezas em relação ao ITRF. No âmbito nacional, será apresentado um trabalho pioneiro realizado em 2010 pela Diretoria de Hidrografia e Navegação (DHN), a fim de calcular o SEP da Baía de Guanabara. Nesta pesquisa detectou-se a diferença de $2,5 \mathrm{~cm}$ entre a redução de sondagem tradicional e aquela obtida por maréGPS. Além disto, é apresentado o projeto Alt-Bat, proposto pelo Comitê de Integração dos Componentes Verticais Terrestre e Marítimos (CICVTM), o qual prevê a adoção do geoide como referência vertical para modelos hidrodinâmicos, fornecendo assim, o SEP em nível nacional para os levantamentos hidrográficos. O presente artigo apresenta as perspectivas e desafios a serem superados pelo Brasil na seção 4. E na seção 5 tem-se as considerações finais acerca desta temática.

\section{SUPERFÍCIES DE REFERÊNCIA VERTICAIS PARA O CÁLCULO DE MODELOS SEP}

Em zonas costeiras diferentes superfícies de referências verticais podem ser empregadas para a determinação do SEP, elas podem ser matemáticas, como o elipsoide de revolução; ou físicas, oriundas da maré, ou do campo de gravidade terrestre. Na Figura 3 observa-se o relacionamento entre estas superfícies envolvendo a determinação da Topografia do Nível Médio do Mar (TNMM). O SEP refere-se à separação entre o DCN e o elipsoide nas estações maregráficas. A linha verde representa o geoide. As linhas onduladas representam o NMM e o DCN e pode-se verificar tendências semelhantes. Isso indica que apesar de estarem relacionados, a separação entre estas superfícies será diferente de um lugar para outro. O modelo hidrodinâmico representa esta diferença. Também está indicada nesta figura a separação entre o geoide e o NMM, denominada de TNMM (FIG, 2014).

Figura 3 - Relacionamento entre o geoide, o elipsoide e o Datum da Carta Náutica.

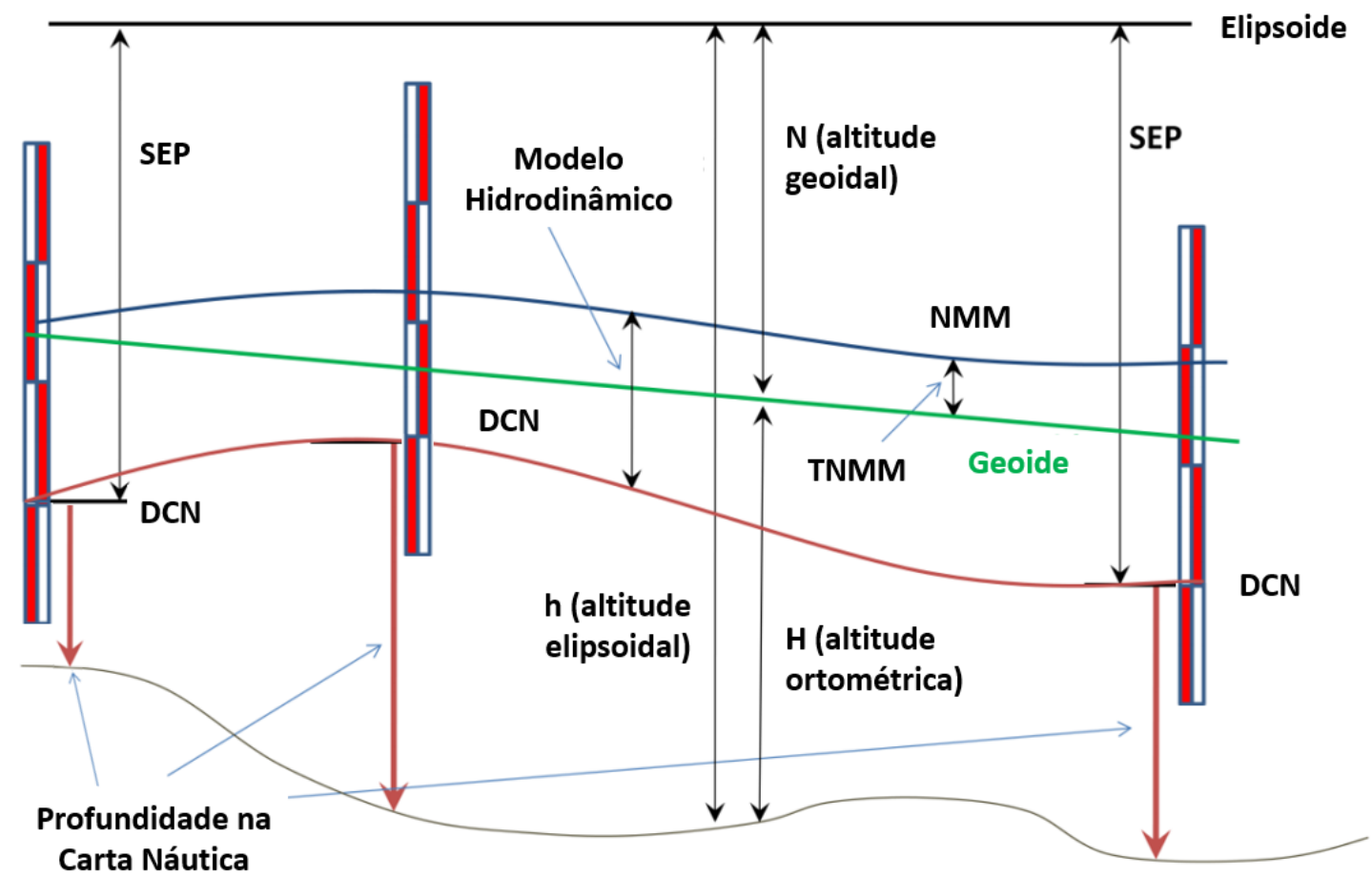

Fonte: Adaptada de DODD e MILLS (2012).

Alguns países do norte europeu, ao invés do NMM, utilizam o geoide como referência vertical dos modelos hidrodinâmicos (ELLMER; GOFFINET, 2006). O Canadá (NUDDS; ROBIN; MACAULY, 2016) e o Reino Unido (ILIFFE et al., 2013) utilizam dados de altimetria satelital e modelos oceânicos calculando a relação entre o referencial vertical oceânico, por exemplo LAT (Lowest Astronomical Tide), e o DCN. Demais métodos e outros detalhes serão vistos na seção 3 . 


\subsection{Superfície elipsoidal}

O Elipsoide de revolução é a superfície matemática, baseada em convenções, representações e cálculos que mais se aproxima da superfície terrestre, sem contar a topografia. E a partir desta superfície, são contadas as altitudes elipsoidais calculadas pelas observáveis transmitidas pelos satélites.

Para garantir uma maior acurácia, o elipsoide precisa estar orientado e fixado em uma determinada época do ITRF, o qual materializa a superfície terrestre a partir de uma série de observações efetuados por diversos sistemas como GNSS, SLR (Solar Laser Range), LLR (Lunar Laser ranger), VLBI (Very Long Baseline Inteferometry) e outros (SIMON, 2013).

Segundo Altamini et al. (2017), a diferença entre o ITRF 2008 e o ITRF 2014, na época, 2010,0 para os eixos x, y e z foi de 1,6 mm, 1,9 mm e 2,4 mm. Assim, as observações GNSS sobre estações maregráficas e referências de nível, promovem altitudes elipsoidais (h) que permitem as transformações entre os sistemas de referência terrestre e oceânicos. (KEYSERS; QUADROS; COLLIER, 2015).

\subsection{Superfície geoidal}

Em uma definição atual, apresentada por Sánchez et al. (2016) e que se baseia nas definições clássicas de Gauss (1876) e Listing (1873), o geoide é dado por $W_{0}$, que é o valor do geopotencial em uma superfície de nível que melhor se aproxima (no sentido dos mínimos quadrados) à superfície do nível médio do mar global, quando este, se encontra totalmente calmo. $O$ valor de $W_{0}$ convencionado para a época 2010,0 e adotado em resolução pela IAG (International Associaton of Geodesy) é de 62.636.853,4 $\mathrm{m}^{2} \mathrm{~s}^{-2}$ com erro formal de $\pm 0,02 \mathrm{~m}^{2} \mathrm{~s}^{-2}$ (IAG, 2015; SÁNCHEZ et al., 2016). A modelagem do campo de gravidade é um tanto laboriosa e leva a uma investigação baseada em levantamentos in situ com gravímetros supercondutores, relativos e/ou absolutos.

Além das determinações in situ, podem ser feitas observações com gravímetros embarcados em aeronaves, navios e em plataformas orbitais (PLAG et al., 2009). Nessa esfera, destacam-se principalmente as contribuições das missões Challenging Mini-satellite Payload (CHAMP), Gravity Recovery and Climate Experiment (GRACE) e Gravity Field and steady state Ocean Circulation Explorer (GOCE) (FLECHTNER; SCHUH; SNEEUW, 2014). Ao longo dos anos, com as missões para modelagem do campo da gravidade, a maior disponibilidade de observações geodésicas terrestres e o desenvolvimento da computação possibilitaram o cálculo de mais de centenas de modelos de Terra, ditos, Modelos Globais do Geopotencial (MGGs), que têm o geoide e o quase-geoide como uma das funcionais do campo da gravidade, como EGM2008 (SÁNCHEZ; SIDERIS, 2017).

\subsection{Superfícies a partir do NMM}

De acordo com Feng, Jin e Zhang (2012), mensurações do nível do mar são realizadas a partir de duas técnicas principais: observações em estações maregráficas e a altimetria por satélites. As estações maregráficas fixas à crosta terrestre medem o nível do mar de maneira pontual, cujas medidas sofrem influência da dinâmica da crosta terrestre, precisando de correções. (FENG; JIN; ZHANG, 2012). Além disto, verifica-se uma baixa distribuição espacial de marégrafos e as observações são efetuadas de maneira relativa ao nível zero do instrumento de medida.

Visando o monitoramento do nível do mar em regiões próximas à costa, em estuários ou baías, PineauGuillou e Dorst (2012) apresentam uma solução que consiste no uso de boias ou navios dotados de receptores GNSS. Analogamente, a publicação FIG (2014) descreve a utilização de marégrafos de fundo, utilizando embarcações com receptores GNSS sob os marégrafos para referenciar suas medições ao elipsoide de referência.

Os produtos derivados de satélites altimétricos, por sua vez, são disponibilizados por diversos institutos de pesquisas. Com o lançamento da missão altimétrica Topex/Poseidon (T/P), em 1992, observações absolutas do nível do mar começaram a ser realizadas diretamente nos oceanos e relacionadas a um elipsoide de referência (SLOBBE; KLEES, 2012). Esta técnica, apesar de possuir perda de precisão na costa devido à 
interação oceano/continente, proporciona uma recobrimento espacial sem precedentes nas observações do nível do mar. Por exemplo, o The National Space Institute at the Technical University of Denmark (DTU Space), instituto dinamarquês de pesquisas vinculado à Universidade Técnica da Dinamarca, gera diversos produtos como: Estimativas da alteração do Nível do Mar, Modelos Globais de Batimetria, Modelos Globais do Campo de Gravidade, Modelos Globais de Marés, Modelo da superfície LAT, Modelos Globais da Superfície Média do Mar e Modelos da Topografia Dinâmica do Mar (DTU, 2020).

Os modelos de superfície derivados de missões altimétricas possuem perda de precisão na costa, conforme citado anteriormente, todavia podem ser corrigidos por modelos hidrodinâmicos. Estes modelos descrevem a reação do corpo d'água introduzindo condições de contorno (batimetria e linha de costa) e forçantes externas (sistema Solar/Lunar e efeitos meteoceanográficos). Ele utiliza um conjunto de algoritmos baseados na dinâmica de fluidos, derivados das leis do movimento de Newton, porém necessitam ser calibrados por estações maregráficas e meteoceanográficas (FIG, 2014; MMA, 2018; DTU, 2020).

Assim, a TNMM, também chamada de Sea Surface Topography (SST), pode ser medida por meio da média do desvio entre a superfície dos oceanos em relação ao geoide, sendo influenciada por diversos fatores meteorológicos e oceanográficos. Pode ser determinada na costa por estações maregráficas onde o nível médio do mar foi observado e nivelado ao referencial altimétrico terrestre; ou nos oceanos, pelos satélites altimétricos, obtido pela diferença entre a Superfície Média do Mar (MSS - Mean Sea Surface) e o geoide, caso em que também é chamada de Topografia Dinâmica Oceânica Média (MDOT - Mean Dynamic Ocean Topography ou MDT - Mean Dynamic Topography) (FIG, 2014).

\subsection{Datum da Carta Náutica}

De acordo com a publicação C-32 do Dicionário Internacional de Hidrografia, o Datum da Carta Náutica é uma superfície permanentemente estável, geralmente a baixa-mar, à qual estão referenciadas as sondagens ou alturas de maré, sendo também chamado de nível de referência ou plano de referência (OHI, 2020c).

Visando a segurança da navegação, a OHI recomenda que, em áreas oceânicas sob influência de maré, a Menor Maré Astronômica (LAT) ou um nível equivalente próximo, seja adotado como DCN para as profundidades. Define-se a LAT como a menor baixa mar prevista para ocorrer sob condições meteorológicas médias e sob qualquer condição astronômica (OHI, 2018). Simon (2013) ressalta que a LAT é um valor aproximado e não é possível calculá-lo de maneira acurada e estável, pois depende da qualidade e da duração dos dados observados, da correção dos efeitos meteorológicos e oceanográficos, do método de cálculo, do tipo e da amplitude das marés. Entretanto uma vez definido, pode ser utilizado como DCN.

Alguns países, como o Canadá, utilizam outros níveis de referência, baseados em critérios astronômicos, como a média em cada ano das menores baixa-mares previstas (LLWLT - Lower Low Water, Large Tide), em um ciclo de 19 anos (CHS, 2013). Já para os Estados Unidos e o Japão, ao invés de previsões de maré, o DCN é baseado na média das mais baixas marés (MLLW - Mean Lower low Water) observadas em um período chamado de Época Nacional dos Dados Maregráficos (NTDE - National Tidal Datum Epoch) (NOAA, 2020a; ROEBER, 2016). Nas cartas náuticas brasileiras, o DCN é conhecido como nível de redução e corresponde aproximadamente à Média das Baixa-mares de Sizígia (MLWS - Mean Low Water Springs) (MARINHA DO BRASIL, 2020).

Na definição do DCN pode ocorrer que o registro da variação da curva de maré em uma única estação não seja suficiente para representar toda a área de um levantamento hidrográfico. Uma alternativa seria realizar o zoneamento de marés, que consiste na interpolação, ou na extrapolação linear dos dados observados entre duas estações maregráficas situadas nos extremos da área, dividindo-a em zonas (DHN, 2017). Entretanto, "dependendo da região este método pode ser impreciso porque assume que as componentes que não são astronômicas, variam no espaço e no tempo da mesma forma que as componentes das marés." (HESS et al., 1999, p.11). Países estrangeiros utilizam outras técnicas, integrando estações maregráficas; modelos hidrodinâmicos e oceânicos globais; modelos geoidais locais e modelos globais do geopotencial para assim, definir o SEP. 


\section{EVOLUÇÃo HISTÓRICA QUANTO ÀS ESTRATÉGIAS PARA CÁLCULOS DE MODELOS SEP}

Constata-se que o cálculo do SEP teve seu início na década de 90, no Canadá, conforme Wells, Kleusberg e Vaniček, (1996). Segundo esses autores, há uma busca na avaliação do uso de uma superfície de referência contínua para visualização, gerenciamento e aquisição de dados hidrográficos no ECDIS, de forma que o sistema possa informar as profundidades em tempo real ao navegante, ao invés da mais baixa situação de maré possível, bem como, as incertezas destas medidas. Outros autores destacam tanto a importância na determinação da TNMM, por meio de modelos hidrodinâmicos e satélites altimétricos, quanto à importância destes modelos para a gestão de processos na zona costeira. (FIG, 2014; ODAMAKI, 2003). Verifica-se assim a necessidade de desenvolvimentos quanto ao SEP, visto que, em junho de 2020, a OHI publicou a Guidelines for creating S-100 Product Specification - S-97, sobre as cartas eletrônicas da classe S-10X, em que as profundidades podem ser informadas em tempo real, em relação a diferentes referenciais verticais (OHI, 2020b).

A evolução histórica, no que se refere às principais estratégias desenvolvidas na definição do SEP, pode ser agrupada em dois períodos distintos: um período de criação, compreendido de 1995 a 2010, e outro de consolidação, compreendido entre 2010 e 2020 (Figura 4).

Nestas duas décadas vários modelos foram desenvolvidos, como o AUSHYDROID, na Austrália (MARTIN; BROADBENT, 2004); o projeto Bathyelli na França, (PINEAU-GUILLOU et al., 2009); na Baía de Concepcion e Golfo de Arauco, no Chile (GONZÁLEZ ACUÑA; ARROYO SUAREZ, 2013); no Rio da Prata, na Argentina (OREIRO; D'ONOFRIO; FIORE, 2016); próximo à fronteira entre Singapura e Malásia (ABDULLAH; OMAR, 2011); no Mar Báltico (BSCD - Baltic Sea Chart Datum) (FAMOS, 2020); no Mar Mediterrâneo (GRGIć, NEREM, BAšIć, 2017); ou até nas proximidades da Ilha Rei George, na Antártida (KE et al., 2020).

Figure 4 - Evolução histórico-científica de modelos SEP no mundo.

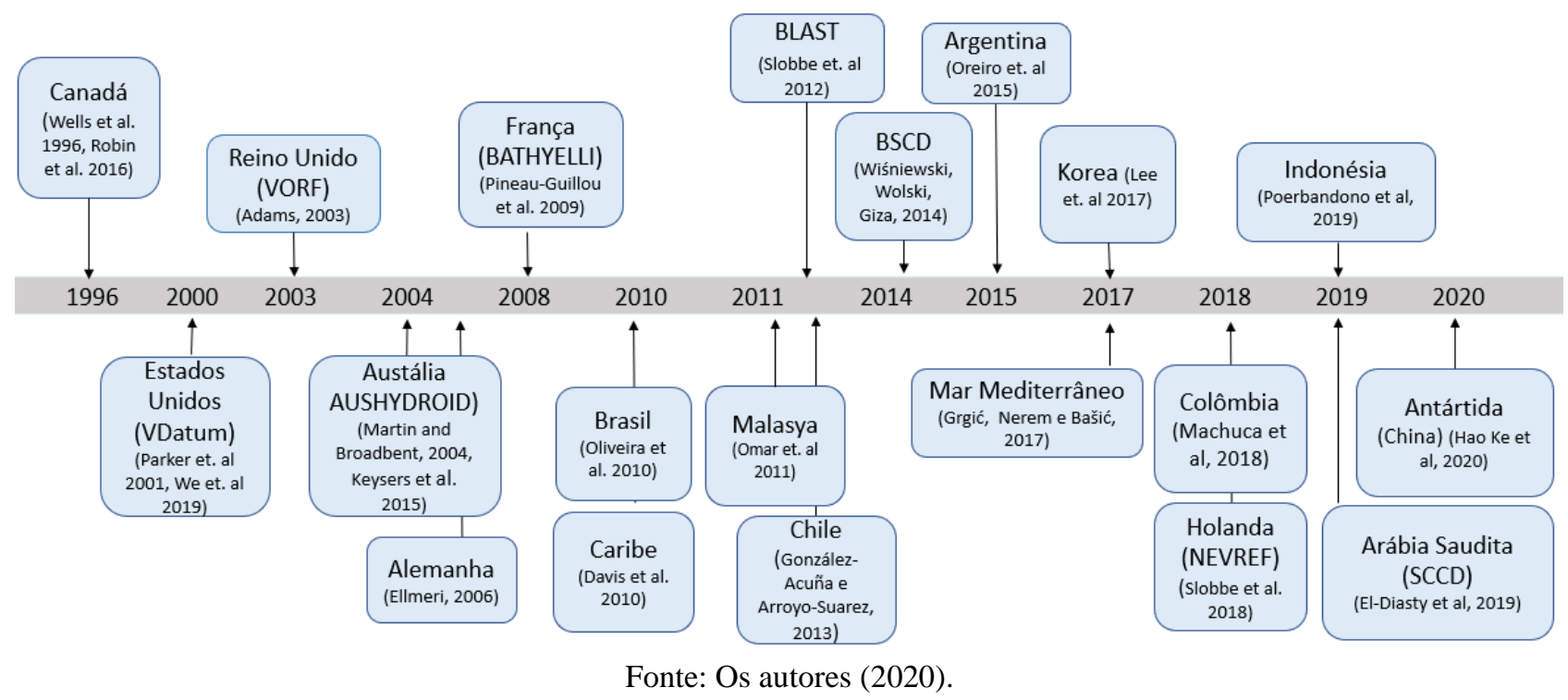

Na seção 3.1 serão apresentadas as estratégias adotadas pelo Canadá, Estados Unidos, Reino Unido, e países do norte europeu, Colômbia e Arábia Saudita e posteriormente a nível nacional na seção 3.2.

\subsection{Panorama Internacional}

\subsubsection{PERÍODO DE 1995 A 2010}

O Canadá foi um dos pioneiros nas pesquisas relacionadas aos modelos de separação entre superfícies de referências verticais, iniciando em 1995, em St. Lawrence, os levantamentos hidrográficos utilizando a maré GPS. Nesta pesquisa foram estabelecidas ao longo do canal uma rede de estações para possibilitar o uso do 
posicionamento RTK, utilizado o software Hypack para navegação em tempo real e medição da maré GPS com as soluções RTK. Assim, foram efetuados levantamentos GNSS em cada estação maregráfica primária, e o SEP foi determinado por meio de uma interpolação linear entre estas estações maregráficas em relação ao Datum Vertical Geodésico Canadense (CGVD28- Canadian Geodetic Vertical Datum of 1928). A validação dos dados da maré GPS foi feita comparando com o modelo hidrodinâmico SPINE (LEFAIVRE et al., 2010)

Nos Estados Unidos da América (EUA), o software VDatum, foi desenvolvido com o objetivo de permitir a conversão entre três tipos de referenciais verticais: marégráficos, elipsoidais e ortométricos. Tratase de um algoritmo de transformação de Data, considerado como traversing minimum spaning tree, no qual cada nó da grade representa um Datum individual. O projeto inicial se deu na Baía de Tampa, na Flórida, com o objetivo de integrar batimetria e topografia. Foi utilizado o modelo hidrodinâmico Princeton Ocean Model com uma série temporal de maré de 18,6 anos e uma grade variando de 100 a $1000 \mathrm{~m}$. Foi alcançado um desvio padrão de $2,7 \mathrm{~cm}$ entre os dados previstos pelo modelo e os observados na região. Nos locais onde os modelos não eram disponíveis, ou totalmente calibrados, como um estudo conduzido na Baía de Delaware, utilizou-se a técnica Constituintes de Marés e Interpolação Residual (TCARI - Tidal Constituent and Residual Interpolation). Esta técnica consistiu na interpolação espacial das constantes de amplitude e fase das componentes harmônicas e dos resíduos provenientes de efeitos não relacionados com a maré, através da solução numérica da equação de Laplace (HESS, 2002; PARKER, 2002; PARKER et al., 2003; NOAA, 2004; NOAA, 2020b).

Após a aplicação do VDatum em projetos locais, como em New York Bight, Puget Sound, Chesapeake Bay, New York Harbor, foi investigada a criação de um banco de dados de batimetria e topografia integrados para todo o território nacional, chegando ao Alaska e o Havaí. Para tal, a estratégia consistia em quatro etapas: os dados de batimetria e linha de costa eram coletados, verificados e gerada uma grade de elementos finitos; aplicava-se as condições de contorno no Modelo avançado de circulação para águas oceânicas, costeiras e estuarinas (ADCIRC - ADvanced CIRCulation Model for Oceanic, Coastal and Estuarine Waters) utilizando computadores do Departamento de Administração Oceânica e Atmosférica Nacional (NOAA - National Oceanic and Atmospheric Administration); os resultados eram refinados através de TCARI; os dados eram enviados ao serviço de Levantamento Geodésicos Nacional (NGS - National Geodetic Survey) para inclusão no software VDatum, com os algoritmos do elipsoide de referência, do resultado do modelo geoidal e da análise da TNMM (MYERS et. al., 2005; MYERS et al., 2007; NOAA 2020b).

No norte europeu, em 2002, o Serviço Hidrográfico Alemão iniciou um projeto para determinar a superfície do DCN da costa do mar Báltico e referenciá-la ao elipsoide do Sistema de Referência Terrestre Europeu de 1989 (ERTF89 - European Terrestrial Reference System 1989). Foram efetuados rastreios GNSS em referências de nível na costa e em águas interiores sob influência de maré. O quase-geoide EGG97 foi utilizado como sistema de referência para determinação do nível do mar, resultando em um modelo de elementos finitos com 32 mil nós. Foi determinado para cada nó o valor das amplitudes de maré, obtendo assim o SEP para cada ponto da grade do modelo (ELLMER; GOFFINET, 2006).

Em 2005, o Serviço Hidrográfico Britânico (UK Hydrographic Office) iniciou o projeto da Rede de Referência Vertical Oceânica (VORF - Vertical Offshore Reference Frame), com o objetivo de calcular modelos de separação entre o elipsoide GRS80, referenciado ao ETRF89 e superfícies de referência verticais na terra e no mar (ADAMS et al., 2006). Segundo Iliffe, Ziebart e Turner (2007), a integração de dados de estações maregráficas e de satélites altimétricos foi realizada por meio da colocação por mínimos quadrados, utilizando como referência vertical o NMM calculado para a época 2000, e dados dos modelos geoidais OSGM02 e OSGM05, nivelamentos, rastreio GNSS, e dados dos modelos hidrodinâmicos. De acordo com Ziebart et al. (2007), o VORF representou uma revolução na indústria naval, uma vez que os levantamentos hidrográficos afastados da costa ("offshore") podiam ser reduzidos sem o auxílio de estações maregráficas, ou de cartas cotidais (Figura 5). 
Figura 5 - Comparação da batimetria utilizando estação maregráfica (esquerda) e utilizando o GPS e o VORF (direita).

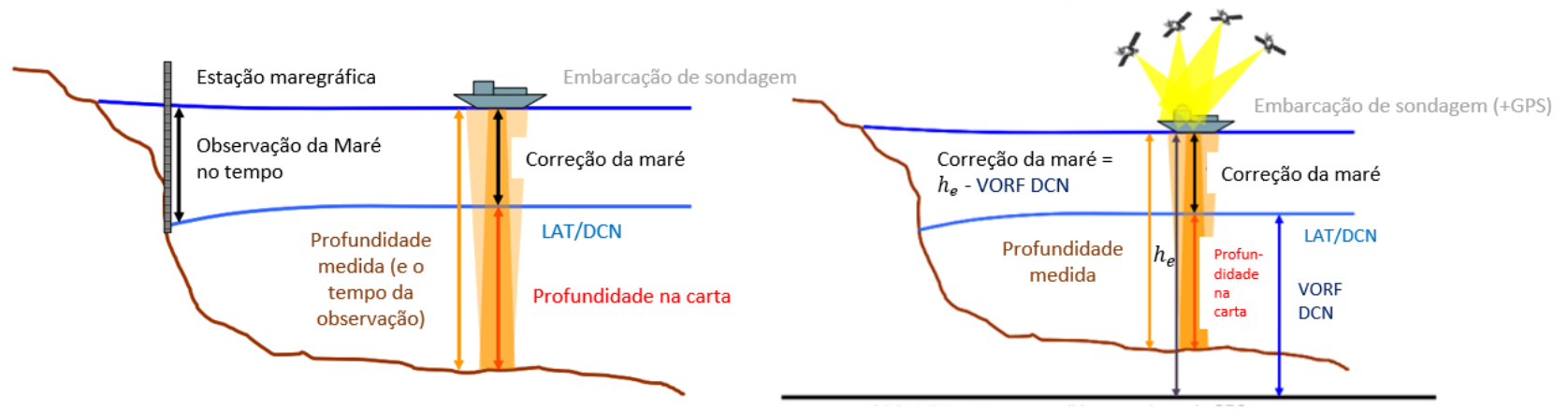

Fonte: Adaptada de ZIEBART et al. (2007).

Em 2009, um conjunto de 14 países deram início ao projeto Bring Land and Sea Together (BLAST), que teve como um de seus objetivos a harmonização do DCN no norte da Europa. Seguindo a metodologia da Alemanha, uma superfície equipotencial do campo de gravidade da terra foi utilizada como referência vertical para o modelo hidrodinâmico. Com isso, teve-se a vantagem de se dispensar as técnicas de interpolação, como utilizadas no VORF, para preencher a falta de dados entre as estações marégraficas e os satélites altimétricos (HUGHES; BINGHAM 2008; SLOBBE et al., 2012, SLOBBE; KLEES; GUNTER, 2014; BOSCH 2016a).

\subsubsection{PERÍODO DE 2010 A 2020}

A partir de 2010, a metodologia do VORF foi implementada combinando dados do modelo hidrodinâmico NISE10 e do Modelo Oceânico Global CSR4.0 com os dados de estações maregráficas na costa do norte da Europa e altimetria por satélite. A interpolação foi efetuada pelo algoritmo Thin Plate Spline (TPS), o que gerou uma melhora de $23 \%$ na modelagem dos erros (TURNER et al. 2010). Posteriormente, o uso de sete modelos oceânicos globais foi avaliado visando a determinação da LAT em relação ao nível do mar, comparando os dados de 7.389 estações maregráficas localizadas no Reino Unido (TURNER et.al. 2013). Verificaram que a $30 \mathrm{~km}$ da costa existia degradação da acurácia dos dados, todavia, com o uso do algoritmo TPS, obteve-se o desvio-padrão de 0,23 m. No mesmo ano, Iliffe et al. (2013) apresentaram a base matemática Eq. (1) necessária para determinar a incerteza espacial do DCN, em relação ao elipsoide de referência no ETRF89 $\left(\sigma_{\mathrm{h}_{\mathrm{DCN}}}^{2}\right)$. Na Equação $1, k_{c}$ representa o termo de degradação calculado em função de $c$, em que $c$ é a distância à linha de costa, e h equivale a altitude da variável representada, em relação ao ETRF89.

$$
\sigma_{\mathrm{h}_{\mathrm{DCN}}}^{2}=\sigma_{h_{L A T}}^{2}+k_{c}^{2} \sigma_{h_{D C N_{0}}}^{2}+k_{c}^{2} \sigma_{h_{L A T_{0}}}^{2}-2 k_{C}^{2} \sigma_{h_{L A T}}^{2}
$$

Nos EUA, o VDatum também se consolidou e evoluiu de uma extensão de 25 para $75 \mathrm{MN}$ da costa (NOAA, 2020b). Georgas, Wen e Zhao (2013), utilizaram, além do TCARI, a interpolação Spline with Barriers no rio Hudson. A NOAA desenvolveu um procedimento para realizar o Zoneamento de Maré Referenciado ao Elipsoide (ERZT - Ellipsoidally Referenced Zoned Tides) em que, a partir da aquisição de dados coletados durante levantamento hidrográfico, foi possível a criação de novos modelos SEP e a validação de outros já existentes (RICE; RILEY, 2011). Shi, Hess e Myers (2013) utilizaram na Baía de Chesapeake o método da interpolação espacial com equações harmônicas de ordens múltiplas para grades desestruturadas, o que permitiu representar no modelo hidrodinâmico regiões complexas como reentrâncias na costa e ilhas. Ainda, Shi e Myers (2016) apresentaram um método de interpolação estatística para determinar a variação espacial da incerteza que minimizou uma função de custo semelhante à assimilação de dados variacionais 3D (3DVAR) ou Optimal Interpolation (OI). Esta função é amplamente utilizada em aplicações meteorológicas e oceanográficas para a integração de dados de modelos e observações. Estes mesmos autores aplicaram esta metodologia nas baías de Chesapeake e Delaware e encontraram resultados melhores em relação à interpolação quando utilizada a equação de Laplace (Tabela 2). 
Tabela 2 - Estudo de caso nas baias de Chesapeake e Delaware com medições em 117 estações maregráficas comparando os dados observados com os dados do modelo. Erros obtidos com a aplicação da interpolação de Laplace e da interpolação estatística.

\begin{tabular}{c|c|c|c}
\hline Resultado/erro & Erro máximo absoluto $(\mathbf{c m})$ & Erro médio absoluto $(\mathbf{c m})$ & RMSE (cm) \\
\hline Erro do modelo & 28,35 & 4,98 & 7,0 \\
C/ Interpolação de Laplace & 12,76 & 2,19 & 3,02 \\
C/ Interpolação Estatística & 7,63 & 1,28 & 1,86 \\
\hline \multicolumn{2}{|r|}{ Fonte: MYERS (2018). }
\end{tabular}

Na Figura 6 observa-se o cálculo da incerteza total propagada de cada Datum de maré em relação a uma determinada época do ITRF, calculados para a Baía de Chesapeake em 2016, bem como, a incerteza máxima propagada para cada região dos Estados Unidos (NOAA, 2020b).

Figura 6 - No diagrama de cima, a incerteza total propagada (MCU - Maximum Cumulative Uncertainty) é apresentada. Ela é definida pela soma da raiz quadrada das incertezas individuais de cada Datum e entre cada transformação de Data, sendo ITRFxx, o elipsoide North American Datum 1983 (NAD83), o Datum ortométrico NAVD88, o Nível médio do mar local, NMML (LMSL - Local mean sea level) e cada Data de Maré (NMM, MHWS e outros). Abaixo, encontra-se o MCU de cada região dos EUA.
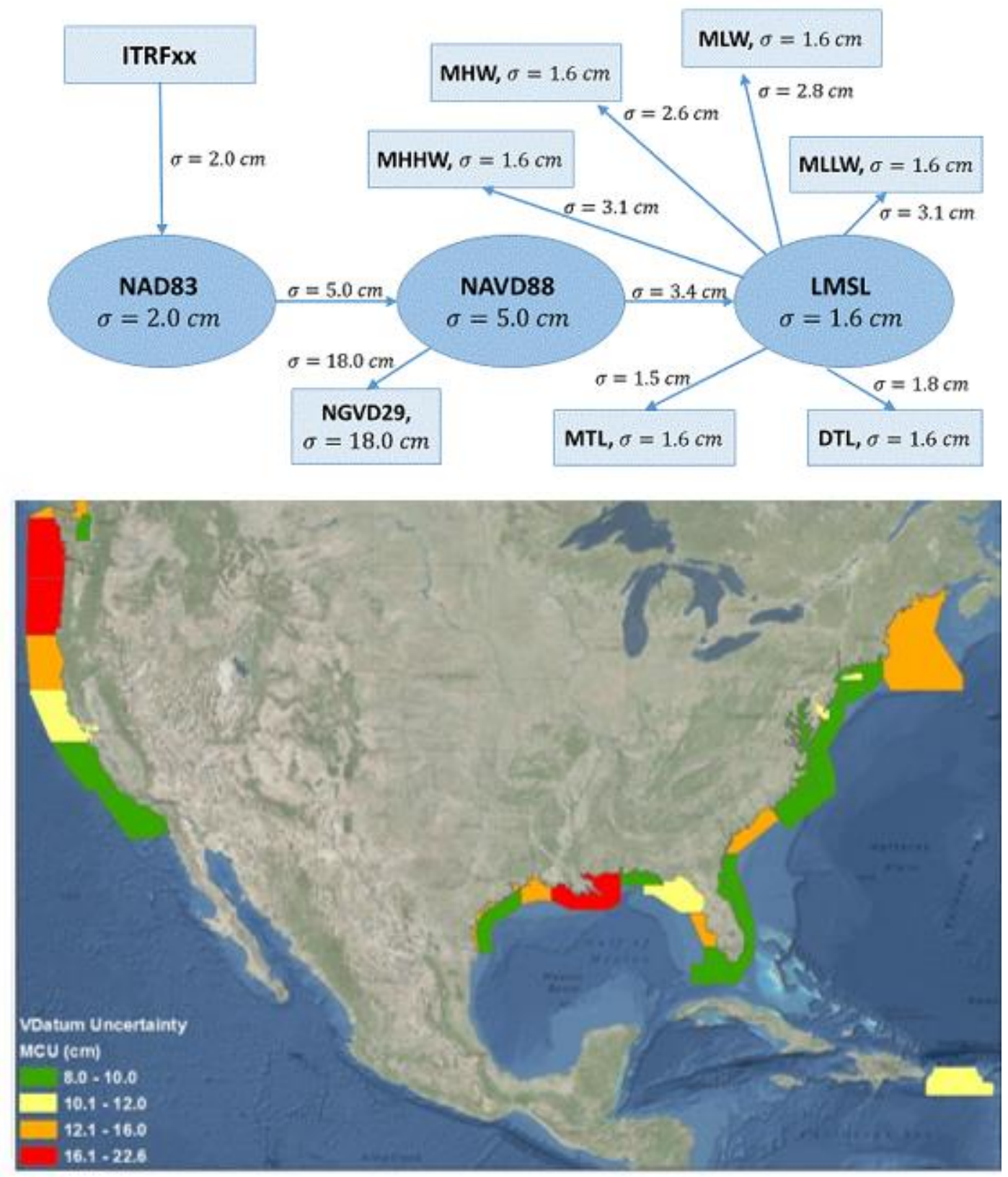

Fonte: MYERS (2018).

Em 2010, Lefaivre et al. (2010) apresentaram a metodologia do projeto The Continuous Vertical 
Datum Canadian Waters Project (Canadá). Neste projeto foi realizado um planejamento de longo prazo, dividido em várias etapas: materialização de pontos de controle, coleta de dados de maré, de GNSS e base de dados para modelos hidrodinâmicos. O país foi dividido em regiões com aspectos hidrográficos específicos (Região Atlântica, Quebec, Ártica e Central, Pacífica). Iniciou-se com trabalhos regionais, como no Golfo de St Lawrence (ROBIN et al., 2012) e Costa de British Columbia (DE LANGE BOOM et al., 2012). Em 2014, foram geradas, para todas as regiões da costa canadense, Superfícies Hidrográficas de Separação Verticais (Hydrographic Vertical Separation Surfaces - HyVSEPs) entre o elipsoide, o DCN e outros sete níveis de maré. Foram combinados como camadas (layers): dados de altimetria por satélite, estações maregráficas, modelos dinâmicos oceânicos (DOM - Dinamic Ocean Model), modelo global oceânico Aviso e modelo geoidal - CGG2013 (com dados dos satélites GRACE e GOCE).

As metodologias do VDatum e do VORF foram conjugadas, utilizando-se um interpolador de Laplace nas grades desestruturadas com resolução de 30 a 100 m, além de um suavizador de elementos finitos (FE Finite Element Smoother). Considerou-se também a variabilidade não linear na interpolação entre os dados de altimetria por satélites e das estações maregráficas. Uma nova metodologia para ajustar o referencial vertical oceânico (LLWLT) aos DCN locais (LLWLT definido para uma época) foi implementada. As incertezas do modelo SEP foram baseadas na soma dos quadrados das incertezas individuais do geoide, do MDT e das medições maregráficas - com base nas formulações de Iliffe (2013) e NOAA (2020b). Foram encontrados valores em relação ao ITRF iguais a 7,5 cm (CANEAST), 6,9 cm (CANWEST), 6,6 cm (CANNORTH) e 17,7 cm (CANHUD) para cada uma das regiões (Figura 7) (ROBIN et al., 2014; ROBIN, 2016).

Figura 7 - Acima são apresentadas as regiões hidrográficas com características específicas e distribuição espacial das estações maregráficas; Abaixo as altitudes elipsoidais do LLWLT.

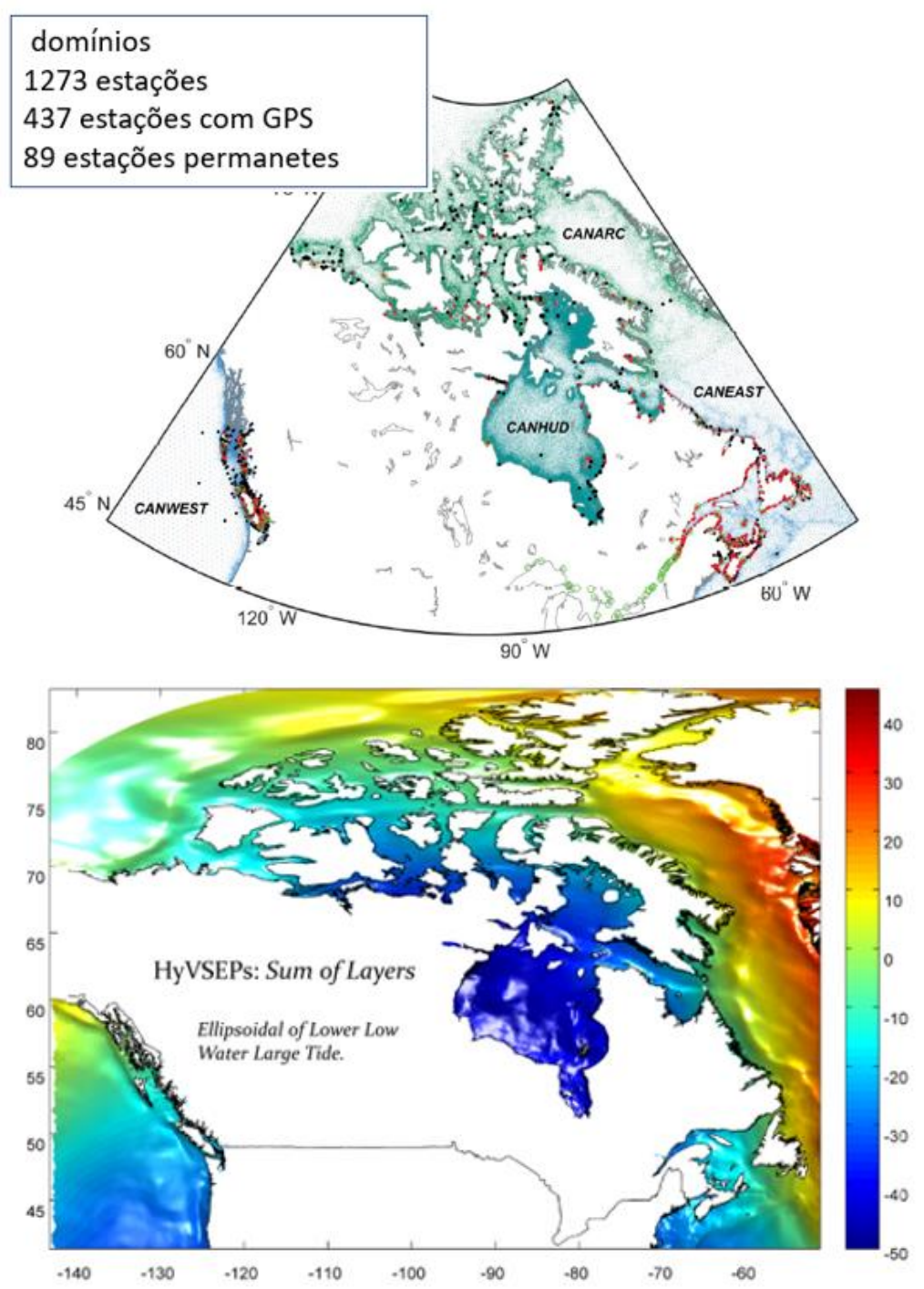

Fonte: ROBIN et al. (2014) e ROBIN et al. (2016). 
No norte europeu, após o projeto BLAST, Holanda e Bélgica lançaram o projeto NEVREF, com o objetivo de calcular a LAT a uma acurácia de $10 \mathrm{~cm}$ em relação ao ITRF, utilizarando o quase-geoide como superfície de referência vertical para o modelo hidrodinâmico. Neste projeto, a LAT e o quase-geoide foram calculados de forma consistente e independente. Consistente, pois para a realização de ambas as superfícies de referência foi utilizado o mesmo modelo hidrodinâmico regional e o mesmo campo de força (marés, vento, pressão do nível do mar, temperatura e salinidade). E independente, porque o modelo hidrodinâmico foi utilizado para determinar a separação entre o quase-geoide e a LAT e também para obter o quase-geoide a partir das medições por altimetria por satélite. Essa dificuldade de referenciar o modelo hidrodinâmico ao quase-geoide e a estimativa do quase-geoide, em si, foi resolvida da seguinte forma: utilizando os valores da LAT modelados nas estações maregráficas e nos modelos hidrodinâmicos, bem como dados de gravimetria e radar altimétrico para aumentar a acurácia do geoide e das correções do MDT. Assim, as altitudes elipsoidais da LAT foram obtidas com a adição das altitudes quase-geoidais aos valores de LAT modelados. (SLOBBE; KLEES; GUNTER, 2014).

Comparando com as outras metodologias de interpolação e calibração de modelos utilizados, Slobbe et al. (2018a) afirmaram que as técnicas Thin Spline e Equações de Laplace, utilizadas por Turner et al. (2010) e Robin et al. (2016), respectivamente, não foram empregadas de maneira ideal. Segundo Slobbe et al. (2018a), a LAT em águas rasas é significativamente alterada por fatores não lineares, assim eles recomendam uma calibração em tempo real e que a validação da LAT, obtida pelo modelo hidrodinâmico, seja efetuada de acordo com Iliffe et al. (2013). Ao se comparar, em termos de RMSE, a variância da LAT obtida pelo modelo hidrodinâmico com as estações maregráficas, foi encontrado um valor total de $15,1 \mathrm{~cm}$.

Além do quase-geoide como referência para o modelo hidrodinâmico, os autores sugerem a utilização do filtro Kalman para o cálculo da LAT. Visto que este filtro é um estimador robusto cujos pesos são atualizados sequencialmente, portanto, ideal para séries longas de dados sujeitas a variações não lineares, como é o caso do nível do mar. Por exemplo, quando comparado com o método tradicional do cálculo da LAT, pela solução linear por mínimos, encontraram valores até $45 \%$ menores no Canal inglês, em termos de RMSE.

Devido ao alto custo para a realização de levantamentos gravimétricos marinhos em um curto prazo, os modelos hidrodinâmicos DNSC06MSS-ZUNOv4 também foram utilizados por Slobbe et al. (2018b) para unir os referenciais verticais entre as ilhas do Mar de Wadden e o continente europeu, com um grid de resolução de $1 / 40^{\circ}(\mathrm{E}-\mathrm{W})$ e $1 / 60^{\circ}(\mathrm{N}-\mathrm{S})$. Eles encontraram uma discrepância entre o previsto e o observado menor do que $1 \mathrm{~cm}$ para $30 \%$ dos marégrafos e menor do que $2 \mathrm{~cm}$, para $60 \%$ dos marégrafos. Ressalta-se que todas as estações maregráficas tinham mais de 18,6 anos de dados.

Continuando a evolução do SEP, El-Diasty, Al-Harbi, Pagiatakis, (2019) desenvolveram o Datum da Carta Contínuo na Arábia Saudita (SCCD - "Saudi continuous chat datum”). Eles, além da definição do DCN em relação ao ITRF, apresentaram também a incerteza espacial desta medida, o que não tinha sido apresentado pela Holanda. Foi criado para o Golfo Pérsico com a resolução de $1 / 16$ graus (até $75 \mathrm{MN}$ da costa) e 1/4 graus (além de 75MN da costa). Empregaram o modelo global oceânico de marés canadense WebTide TIN model, o modelo geoidal EGM08, Modelos da Topografia Dinâmica do Mar (MDT15DTU) e 114 marégrafos na costa, com no mínimo 1 ano de observação cada. A incerteza do modelo SEP foi medida pela soma das variâncias do geoide, do MDT e do ajuste do LAT ao DCN, utilizando interpolação kriging. Encontraram valores finais entre $11 \mathrm{~cm}$ e $16 \mathrm{~cm}$ (Figura 8). 
Figura 8 - Modelo final de separação DCN -WGS84; (esquerda) e incerteza final do modelo (direita).
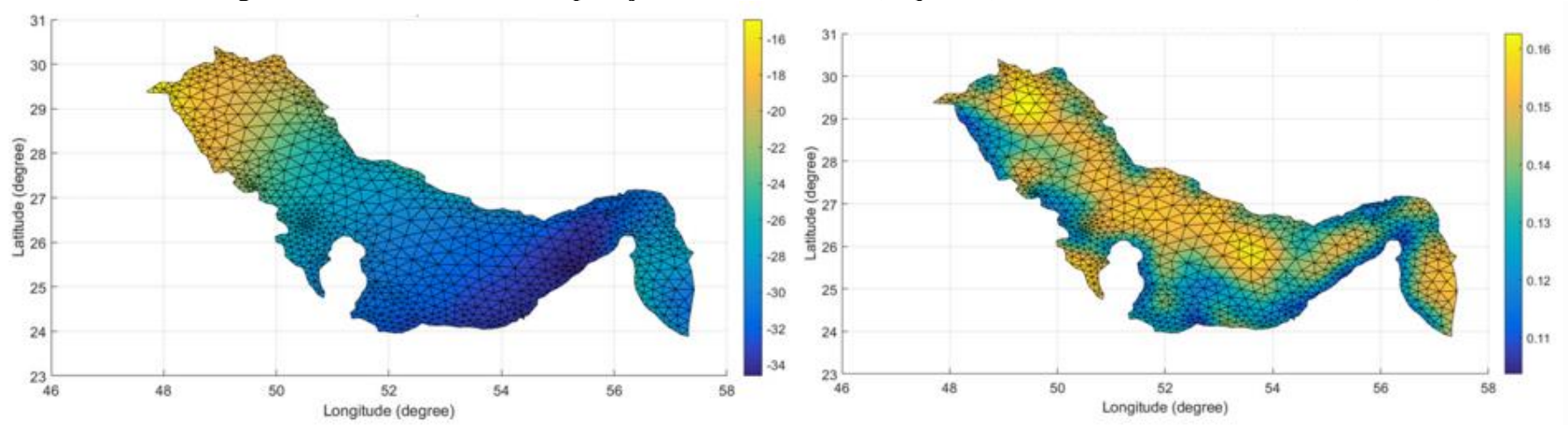

Fonte: EL-DIASTY, AL-HARBI e PAGIATAKIS (2019).

Além da utilização de modelos hidrodinâmicos e oceânicos globais, o SEP também pode ser determinado utilizando somente dados de estações maregráficas, rastreio GNSS e modelos geoidais. Por exemplo, na Colômbia, nas Baías de Málaga e Bueanaventura, com o objetivo de calcular uma superfície hidrográfica de referência vertical (SHRV) foi estabelecida uma rede geodésica de primeira ordem. Esta rede era composta de 11 pontos proporcionando apoio para o controle vertical dos dados do nível da água de 5 estações maregráficas. Com o emprego de embarcações, foram acrescentados outros 200 pontos por meio de observações GNSS-RTK, essas referenciadas ao zero da estação maregráfica mais próxima (Figura 9).

Para as áreas rasas onde o levantamento RTK não era viável, devido à dificuldade na medição dos níveis de água, novas alturas foram geradas com a medição da separação entre Datum de maré e o geoide GEOCOL2004. Para o caso das Baías, os dados foram extrapolados e utilizada a mesma técnica de interpolação espacial por meio do geoide para obter uma cobertura total da área. Com uma resolução de $500 \mathrm{~m}$, a incerteza vertical total da SHRV foi calculada por meio do somatório da grade de levantamentos das altitudes elipsoidais na superfície da água, da materialização da rede geodésica e do controle vertical do nivelamento trigonométrico, estimando um valor final de $\pm 6 \mathrm{~cm}$. Ao comparar a altitude elipsoidal dos Data de nível do mar obtido por nivelamento trigonométrico e por GNSS-RTK, foi encontrada uma médias das diferenças de 5 cm com variação entre -6 à 9 cm. (ALVAREZ MACHUCA et al., 2018).

Figura 9 - Pontos do levantamento GNSS (azul) e vértices rede geodésica de primeira ordem (vermelho) (Figura 9a); Modelo SEP da LAT em relação ao WGS-94 (Figura 9b).

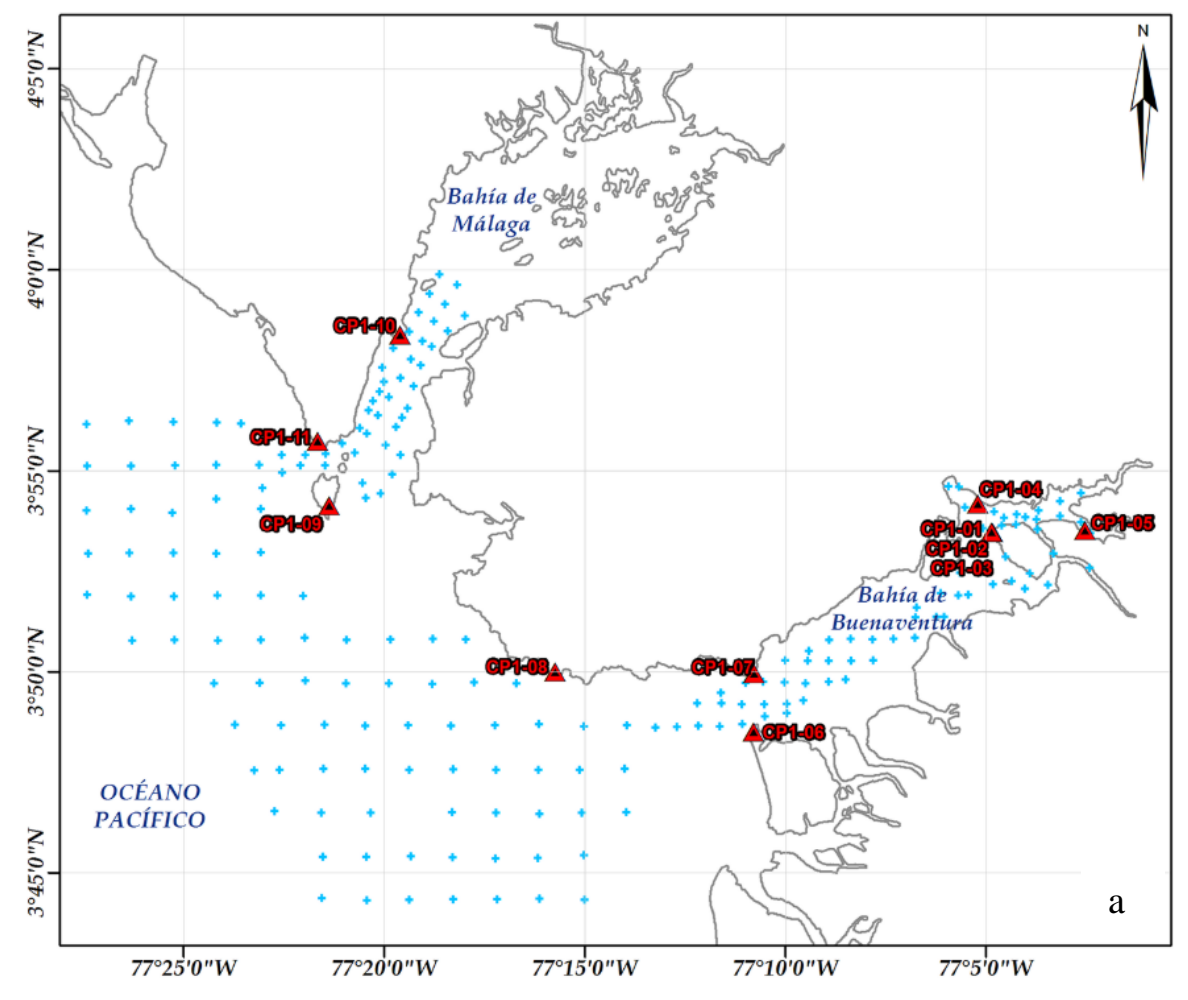




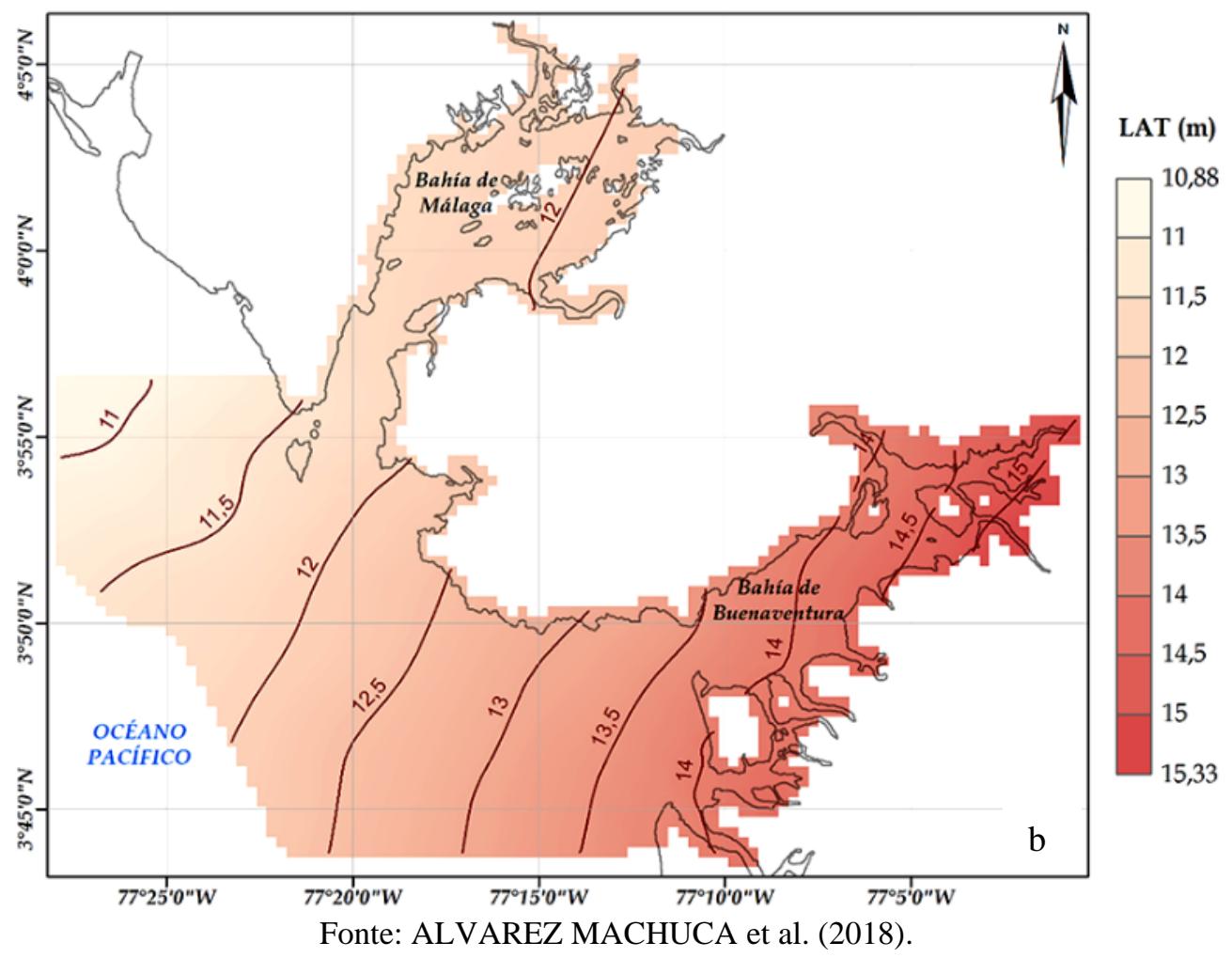

\subsection{Panorama Nacional}

\subsubsection{PERÍODO DE 2007 A 2010}

No Brasil, em 2007, foi realizado na Baía de Guanabara, Rio de Janeiro, um trabalho piloto com o objetivo de conduzir um levantamento hidrográfico com maré-GPS. A área compreendeu a região entre as estações maregráficas da Ponta da Armação e Ilha Fiscal, o que garantiu um bom conhecimento do NR e permitiu considerá-lo constante devido à diferença de $2,3 \mathrm{~cm}$ entre os valores de $\mathrm{Z}_{0}$ (separação entre o NMM local e o DCN) das estações maregráficas (RAMOS, 2007).

Arentz (2009) iniciou no estuário do Amazonas a avaliação do uso de modelos hidrodinâmicos como auxílio à navegação, fruto de um convênio entre a Marinha do Brasil e a COPPE/UFRJ. Os resultados encontrados nos dados batimétricos reduzidos mostraram que nesta pequena área a LAT e o NR eram coincidentes. Ao efetuar a comparação do método do zoneamento de marés, visto em DHN (2017), com a modelagem realizada, foi encontrada uma diferença em termos absolutos de 1,3 m.

Em continuidade ao trabalho de Ramos (2007), Oliveira Junior et al. (2010) realizaram um outro levantamento hidrográfico na Baía de Guanabara, fruto de uma parceria entre a DHN e o Naval Oceanographic Office (NAVOCEANO). Foi utilizado um SEP real para toda a Baía, gerado a partir do modelo hidrodinâmico SISBAHIA (Sistema Base de Hidrodinâmica Ambiental), da Fundação Universidade Federal do Rio de Janeiro, e o MAPGEO 2010 (MATOS et al., 2012). Nesta metodologia foi calculado em seis estações maregráficas a diferença entre o NMML (nível médio do mar local) e o geoide, chamada de resíduos ( $\mathrm{h}_{\mathrm{NMML}}$ N). Em seguida uma grade de valores dos resíduos foi gerada utilizando uma interpolação polinomial de primeira ordem. Esta grade foi somada ao geoide e assim se obteve o NMML para a região. As altitudes elipsoidais (h_RN) das estações maregráficas foram determinadas com o pós-processamento dos dados GPS coletados. A altitude elipsoidal do NMML (h_NMML) foi calculada adicionando o valor de h_RN à distância de cada referência de nível ao NMM. As altitudes geoidais $(\mathrm{N})$ foram fornecidas pelo MAPGEO2010. (Figura 10). 
Figura 10 - Domínio do modelo hidrodinâmico (Figura 10a). Com os valores da altitude geoidal (N) para cada referência de nível foi possível gerar os resíduos do geoide. (Resíduos $=\mathrm{h}_{\mathrm{NMML}-\mathrm{N}}$ ) (Figura 10b).

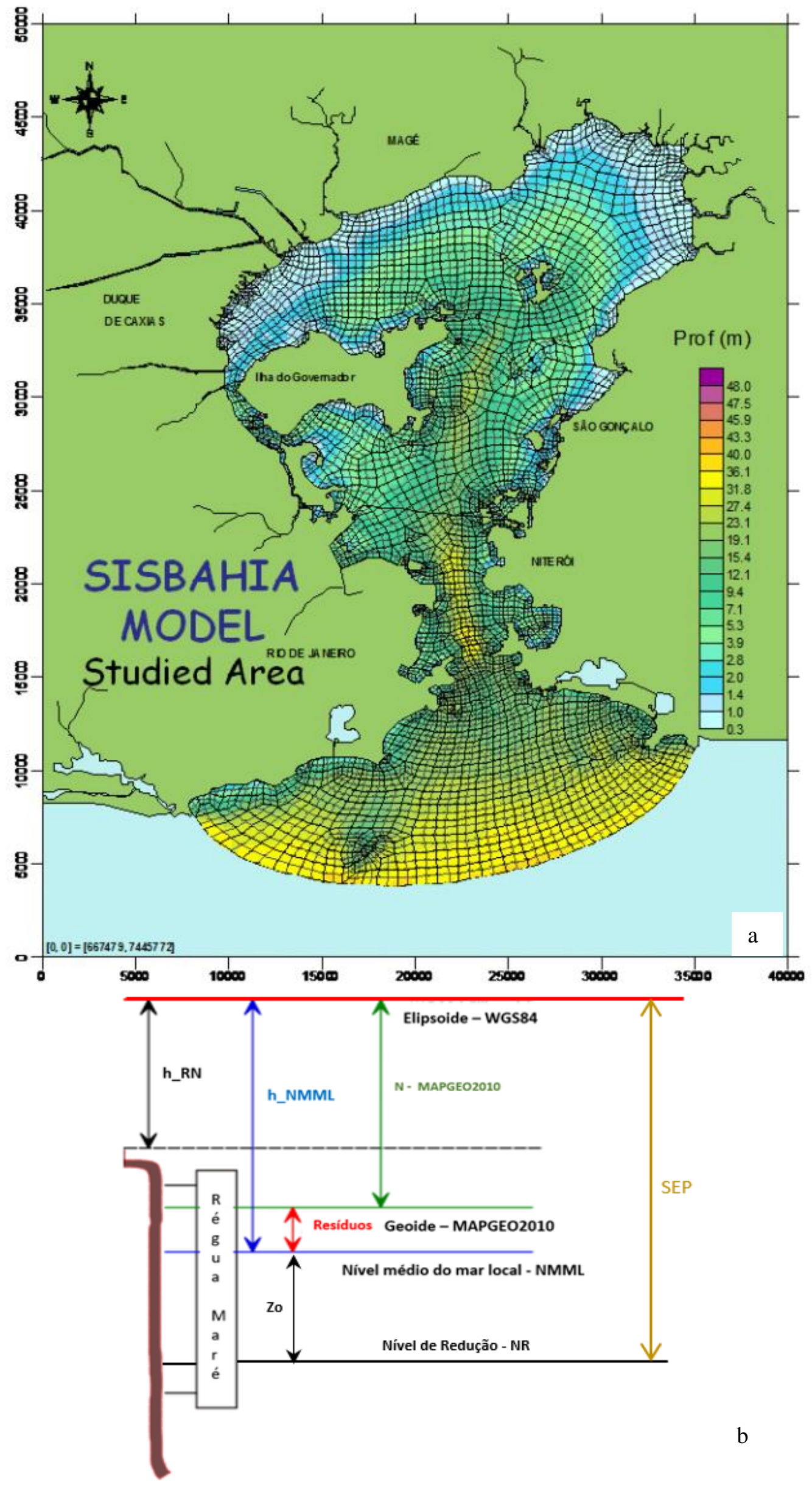

Fonte: OLIVEIRA JUNIOR et al. (2010). 
O modelo final de separação (SEP) entre o elipsoide e o DCN foi produzido com uso da Eq. (2). Em que $Z_{0}$ representa a separação do NMML ao NR, obtido para cada estação maregráfica e cada nó da grade do modelo hidrodinâmico (Figura 11):

$$
\mathrm{SEP}=N+Z_{0}+\text { Resíduos }
$$

Figura $11-Z_{0}$ : Variações do Datum da Carta Náutica ao longo da área de sondagem; N: Altitudes Geoidais obtidas pelo MAPGEO2010 (metros); Resíduos: NMML - Geoide MAPGEO2010 (metros) resultado das seis estações maregráficas utilizadas; SEP: Modelo de Separação (SEP) entre o DCN e o elipsoide.
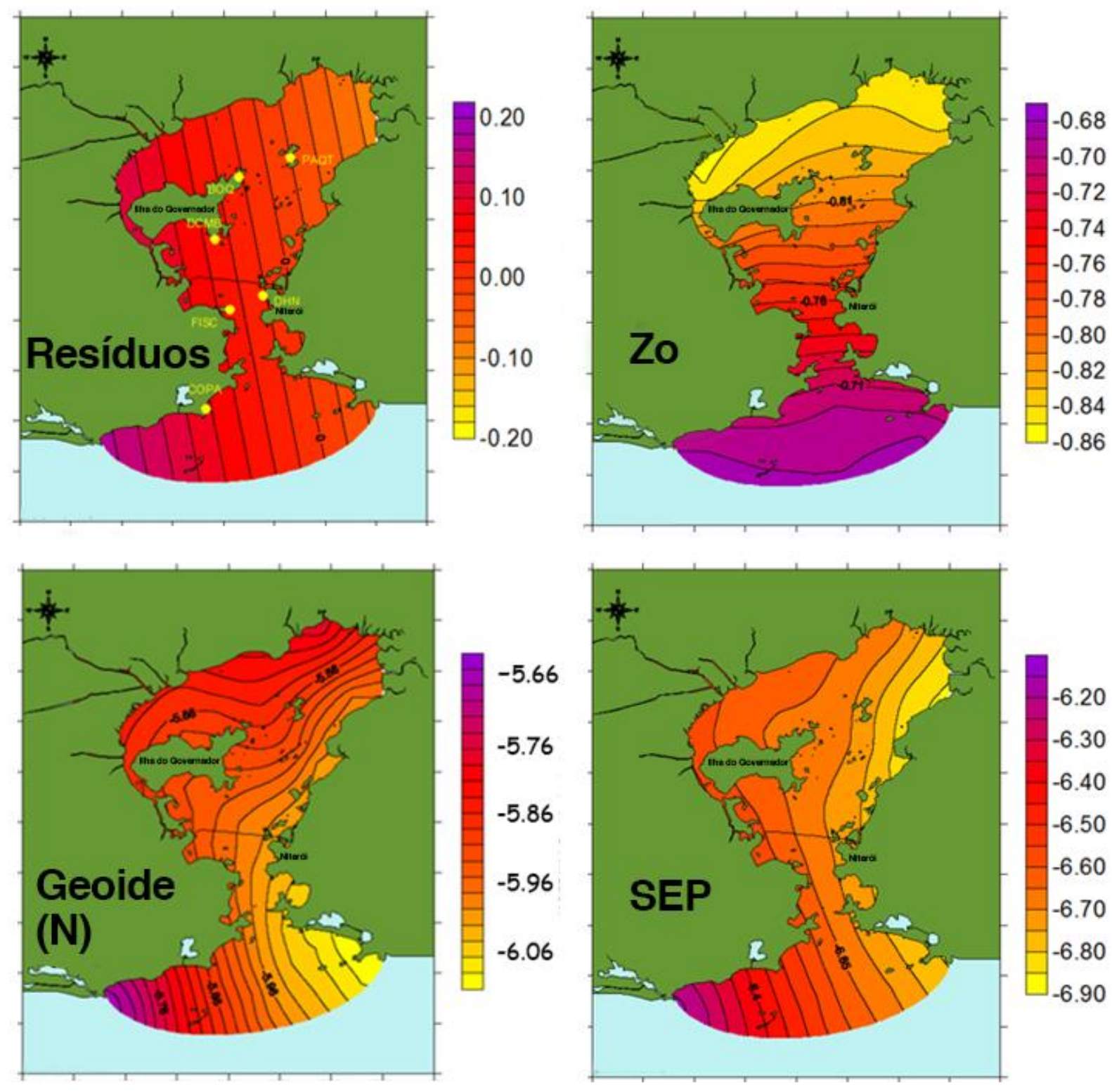

Fonte: OLIVEIRA JUNIOR et al. (2010).

Ao comparar as superfícies batimétricas, criadas utilizando o método tradicional de marés e maré-GPS (PPP), foi encontrada uma diferença média de $2,5 \mathrm{~cm}$, com um desvio padrão de $5,1 \mathrm{~cm}$. A comparação do modelo hidrodinâmico ADCIRC com o SISBAHIA resultou em diferenças menores do que $5 \mathrm{~cm}$ na região de estudo. 
Em 2015 foi dado um primeiro passo para a comunicação de diferentes instituições na direção da vinculação entre setores de topografia e hidrografia, quanto aos referenciais verticais em zonas costeiras, com a criação do Comitê de Integração dos Componentes Verticais Terrestre e Marítima (CICVTM), pela Comissão Nacional de Cartografia (CONCAR). A realização de um ou dois estudos pilotos na costa brasileira também foi abordada, bem como, a necessidade de capacitação técnica para a condução dos trabalhos e a questão do financiamento dos projetos no âmbito da integração das componentes verticais (CONCAR, 2016).

Como conclusão dos trabalhos do CICVTM, duas propostas de unificação dos referencias verticais foram apresentadas por Bosch (2016c). A primeira adotando o NMM como referência para modelos hidrodinâmicos, utilizada no VORF; e a segunda, adotando uma superfície vertical estável, como o quasegeoide, utilizada no BLAST. A resposta se deu por meio do Programa Nacional para a Conservação da Linha de Costa, (PROCOSTA), proposto pelo Ministério do Meio Ambiente, em 2018, tendo como um dos subprojetos o Alt-Bat cujo objetivo é "a integração dos níveis de referência altimétricos e batimétricos e a determinação da cota de inundação como interseção entre o nível d'água máximo projetado e a superficie topográfica" e segue a linha dos projetos BLAST, utilizando o geoide como referência vertical para os modelos hidrodinâmicos (LUZ, 2018).

A metodologia indica a utilização de dados de gravimetria terrestre, marinha, aérea e dados de modelos geopotenciais globais, visando a realização de um processamento preliminar e cálculo de um geoide provisório. Em seguida, utilizam-se as informações de altimetria satelital para o refinamento do geoide (por meio do desvio da vertical). Os dados na costa serão corrigidos por meio de modelos hidrodinâmicos regionais referenciados ao geoide, com a vinculação das estações maregráficas às estações GNSS. Assim serão gerados produtos como um geoide refinado. Posteriormente, utilizam-se dados do NMM, do controle geodésico das estações maregráficas (CGEM), das altitudes das referências de nível (RRNN), e do geoide (N), visando calcular o NMM em relação ao elipsoide que, mediante análise harmônica, possibilitará o cálculo da LAT, bem como, de outras superfícies (LUZ, 2018).

Ações preliminares do Alt-Bat foram tomadas pelo Instituo Brasileiro de Geografia e Estatística (IBGE), como a materialização de uma Rede Geodésica de Referência Costeira (RGRC), para preencher a lacuna de dados entre as referências de nível das estações maregráficas na costa entre as cidades de Macaé e Niterói, onde foram instaladas 500 referências de nível, representando um importante início para a modelagem do SEP das cartas náuticas (SOARES et al., 2019).

Outro estudo foi efetuado por Nascimento (2019), que comparou os dados de ALB (Airborne LASER Bathymetry Lidar) com a batimetria multifeixe na região de Fernando de Noronha. Utilizando os dados da altitude elipsoidal de somente uma referência de nível, encontrou um desvio padrão das diferenças de 0,289 $\mathrm{m}$, com intervalo de confiança de $95 \%$. Um dos motivos para tal incerteza foi atribuída à modelagem do SEP.

Um ano depois, Santana (2020) utilizou, de forma pioneira, modelos globais de LAT em relação ao elipsoide, derivado de altimetria satelital. Em estudo realizado em estações maregráficas de Fortaleza e Imbituba, encontrou um valor $15,6 \mathrm{~cm}$ e $6 \mathrm{~mm}$ acima dos NR das estações maregráficas. Foi pontuado pelo autor que o motivo de tais inconsistências em comparação com observações locais, foi devido à modelagem em áreas costeiras. Além disso, ressaltou a importância de uma maior densificação de estações maregráficas na costa com posições geocêntricas conhecidas para unificação dos referenciais terrestres e oceânicos.

\section{PERSPECTIVAS E DESAFIOS PARA HIDROGRAFIA BRASILEIRA}

No Brasil, a realização do projeto Alt-Bat terá como consequência um modelo SEP a nível nacional para levantamentos hidrográficos com maré-GNSS. Já no contexto global, observa-se uma tendência do crescimento do uso de inteligência artificial na hidrografia e na navegação. Os chamados MASS (Navios marinhos Autônomas de Superfície - Marine Autonomous Surface Ships) já são realidade em alguns países como a Noruega. Os primeiros testes foram realizados em 2019, descortinando um novo horizonte para a navegação, mais segura e sustentável, trazendo inúmeros benefícios, como a minimização de erros humanos e a redução de emissão de gases poluentes (LI; FUNG, 2019). Algoritmos como machine e deep learning permitem identificar as áreas de risco que devem ser evitadas pelos MASS, todavia, assim como um modelo matemático precisa ser validado, os algoritmos precisam ser treinados a partir de observações, fazendo com 
que os dados acurados de batimetria sejam cada vez mais necessários e valiosos. Além disso, o fato destes dados estarem referenciados ao elipsoide GRS80, orientado e fixado a uma determinada época do ITRF, facilita a integração, tanto no espaço quanto no tempo (ADAMS, 2006; HAINS, 2019).

Esta pode ser uma perspectiva para a hidrografia brasileira, entretanto, um grande desafio nacional precisa ser superado: a carência de uma infraestrutura de dados maregráficos, geodésicos e gravimétricos consistentes para a calibração dos modelos em áreas críticas, onde são necessários o gerenciamento costeiro e a segurança da navegação (SANTANA; DALAZOANA, 2020). Bosch (2016b) apresenta um diagnóstico sobre a situação do Brasil e as medidas que podem ser tomadas para integração dos referenciais verticais terrestres e oceânicos a longo prazo, corroborando com os autores O'reilly, Parsons e Langelier (1996) ao afirmarem que "superfícies de referências físicas sempre vão precisar de revisão e o inventário de dados hidrográficos irá crescer significativamente no futuro". Assim, com modelos hidrodinâmicos calibrados, modelos geoidais acurados e superfície terrestre densamente monitorada, gera-se a base necessária para a criação de modelos SEP com um reduzido grau de incerteza, cujos benefícios vão muito além da navegação e da batimetria. Como afirmaram O'reilly, Parsons e Langelier (1996, p. 8):

Apesar do foco inicial dos modelos de separação vertical entre superfícies ter sido direcionado às superfícies hidrográficas (preamar e baixamar), visando os levantamentos e a navegação com cartas eletrônicas que informam a profundidade em tempo real, outros serviços como construção civil, dragagem, gerenciamento costeiro, necessitarão eventualmente, de tais modelos.

Por exemplo, o Ministério do Meio Ambiente lançou o PROCOSTA visando "à modelagem dos processos envolvidos na morfodinâmica costeira, à correta avaliação dos riscos de inundações costeiras e ao desenvolvimento das respectivas estratégias de adaptação e mitigação a tais eventos" (MMA, 2018). Contudo, o projeto Alt-Bat precisará de uma grande quantidade de investimentos para a aquisição de dados visando esta modelagem. Neste sentido, valem serem mencionadas algumas iniciativas de instituições que podem subsidiar tais perspectivas.

Em 2018, a COPPE/UFRJ apresentou o projeto Baías do Brasil, o qual disponibiliza de forma on-line uma base de dados do modelo SiSBAHIA para diferentes localidades, juntamente com seus relatórios descritivos, sendo já disponível para Baía de Guanabara (RJ), Baía de São Marcos (MA), Baía da Babitonga (SC), Baías de Ilha Grande e Sepetiba (RJ), Estuário do Rio Paraíba do Norte (PB), Sistema Lagunar MaricáGuarapina (RJ), Lagoa dos Patos (RS) e Porto do Açu (RJ). (COPPE, 2020).

No mesmo ano, por iniciativa do Centro de Estudo do Mar (CEM) da UFPR, foi implementado o Brazilian Sea Observatory (BSO) para as regiões de Santa Catarina e Paraná, com uma resolução variando de $120 \mathrm{~m}$ a 1/12 $2^{\circ}$. O sistema utiliza o modelo hidrodinâmico MOHID, com código aberto e tem perspectivas para ser acoplado com outros modelos hidrodinâmicos locais, como no Estuário do Amazonas (FRANZ et al., 2018).

Em 2019, o Centro de Hidrografia da Marinha (CHM) passou a utilizar os modelos SWAN para propagação de ondas em áreas costeiras e os modelos hidrodinâmicos ADCIRC, com uma resolução de $100 \mathrm{~m}$ a $4 \mathrm{~km}$ e transporte em duas e três dimensões, tendo já sido gerados modelos na Baía de Guanabara, Baías de Ilha Grande e Sepetiba, Canal de São Sebastião e Ilha Bela (SILVA, 2019).

Cita-se ainda a incorporação do Navio de Pesquisa Hidroceanográfico Vital de Oliveira à Marinha do Brasil, o qual possui o gravímetro dentre os 28 equipamentos científicos instalados a bordo, bem como o uso de VANTS, anunciado pela Companhia de Pesquisa de Recursos Minerais (CPRM), no suporte aos projetos voltados ao monitoramento de toda a costa brasileira e plataforma continental rasa adjacente para a definição da Linha de Costa (CPRM, 2020).

Quanto à aquisição de dados no ambiente costeiro, é de extrema importância avaliar o melhor método, que pode ser por Satélite SAR, levantamento Lidar, batimetrias monofeixe ou multifeixe. Neste sentido, vale citar EMOdnet (2016) e Colin (2016), que apresentam o algoritmo Coastal Mapping Planner (CMP), que emprega a lógica Fuzzy para determinar o levantamento mais adequado de acordo com a profundidade, a transparência da água e o equipamento a ser utilizado.

$\mathrm{Na}$ fase de planejamento, é necessário também analisar a modelagem do SEP antes da coleta dos dados 
referenciados ao ITRF, levando em consideração a incerteza espacial do modelo geoidal, da interpolação da maré, da determinação da TNMM e realizar as correções dos sistemas de maré permanente, dependendo da ordem do levantamento que se deseja atingir. Para o planejamento do SEP em nível nacional, sugere-se consultar Wells, Kleusberg e Vaníčeck (1996), que propõem os detalhes que devem ser levados em consideração para o seu desenvolvimento, como acurácia desejada, resolução do modelo, cobertura, recursos disponíveis, redes de estações maregráficas e GNSS, armazenamento e atualização do banco de dados, dentre outros.

Assim, com uma infraestrutura de dados consistentes, a perspectiva é de um ciclo virtuoso para a economia do país, com uma maior acurácia dos modelos SEP e menor incerteza dos levantamentos (Figura 12). Essas etapas do ciclo se mostram essenciais em áreas onde a folga abaixo da quilha é medida em tempo real, elevando a movimentação de navios em áreas portuárias com mais segurança e eficiência, cita-se, por exemplo, o sistema ReDRAFT, criado pela Argonáutica em parceria com o Tanque de Provas Numéricos da Universidade de São Paulo (TPN-USP), e a Praticagem de Santos. O sistema avalia as condições ambientais de forma que o calado máximo considerado para as manobras dos navios não seja fixo, e sim possa variar de acordo com as características da embarcação, do tipo de manobra e das condições meteoceanográficas. Em Santos, onde foi implantado, o tempo de fechamento do porto por restrições do calado era de oito dias em 2013, já em 2017 foi reduzido para apenas dois dias e cresceu 24\% o número de manobras com calado acima de $12 \mathrm{~m}$. Maior calado representa maior movimentação de carga no porto, pois o aumento de $10 \mathrm{~cm}$ proporciona mais mil toneladas, ou 65 contêineres para o transporte (CONAPRA, 2020a; CONAPRA, 2020b; ARGONAUTICA, 2020).

Figura 2 - Círculo virtuoso sobre a importância de uma infraestrutura de dados para modernização do porto e desenvolvimento da economia do país.

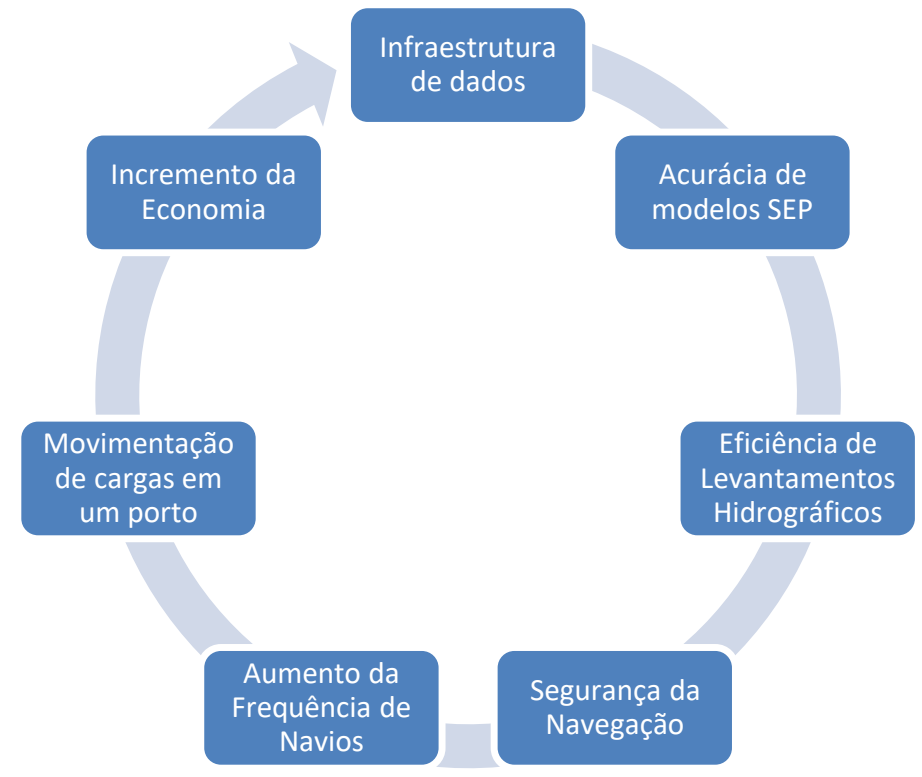

Fonte: Baseado em Ziebart et al. (2007) e GERGS (2019).

A este ponto, percebe-se que o início do ciclo é uma infraestruturua sólida de dados ambientais, terrestres e oceânicos. Portanto, valem ser feitos alguns questionamentos para nortear futuros trabalhos visando a definição do DCN com uma acurácia de $10 \mathrm{~cm}$ em relação ao ITRF.

Desafios quanto aos dados maregráficos e modelagem:

a) Iliffe (2013) apresentou a base matemática para integrar dados de altimetria satelital e estações maregráficas, chegando a uma incerteza de $10 \mathrm{~cm}$ nas proximidades da costa. Em um curto prazo, tal metodologia poderia ser aplicada ao Brasil com tal acurácia? Se não, quanto seria preciso melhorar a infraestrutura de dados?

b) El-Diasty (2019) utilizou modelos globais de marés, modelo geopotencial global para calcular o DCN em relação ao ITRF com uma acurácia de entre 0,11 e $0,16 \mathrm{~cm}$. Em um curto prazo, tal 
metodologia poderia ser aplicada ao Brasil com tal acurácia? Se não, quanto seria preciso melhorar a infraestrutura de dados para validar estes modelos?

c) No projeto NEVREF, Slobbe (2018) utilizou o quase-geoide como referência vertical para o modelo hidrodinâmico e cálculo da LAT, unindo os referencias verticais entre ilhas com uma acurácia sub-centimétrica. Em um longo prazo, quais dados seriam necessários para aplicar tal metodologia no Brasil visando obter esta acurácia? Para mais detalhes pode sobre um roteiro a seguir, pode-se consultar Slobbe (2013b);

d) No projeto VDatum, Shi e Myers (2016) aplicaram na Baía de Chesapeake a interpolação estatística da variação espacial da incerteza para a determinação do DCN. Foi obtido um RMSE de $1,86 \mathrm{~cm}$ entre o observado nas estações maregráficas e o previsto pelo modelo hidrodinâmico. Quantas novas estações maregráficas seriam necessárias para definir o SEP de todos os estuários do Brasil com esta acurácia?

e) Quais recursos financeiros e humanos seriam necessários para instalação destas estações, mantê-las de forma permanente, realizar a manutenção necessária de acordo com IBGE (2017) e disponibilizar os dados de forma on-line para a comunidade?

f) Qual seria o retorno socioeconômico deste investimento em dez anos? Para detalhes dos benefícios, pode ser consultado NOAA (2001).

g) No Canadá foi feito um planejamento de longo prazo para a definição de modelos SEP, dividindo o país em regiões hidrográficas específicas. No Brasil, quais poderiam ser estas regiões? Quais áreas seriam prioritárias para a geração de uma base de dados?

h) A OHI recomenda que a Menor Maré Astronômica (LAT), ou um nível equivalente próximo, seja adotado como DCN em áreas sujeitas a ações de maré. Slobbe (2013) encontrou profundidades mais baixas do que a LAT e do que o LLWLT no canal Inglês e sugere que seja adotado como DCN um valor percentual abaixo da LAT, definido a partir de observações em estações maregráficas durante 18,6 anos. Se o Brasil almejar alterar o tipo de DCN, qual deveria ser este percentual para as cartas náuticas brasileiras?

i) De acordo com Slobbe et al. (2018a), a LAT em águas rasas é significativamente alterada por fatores não lineares. Como realizar uma calibração em tempo real de um modelo hidrodinâmico e gerar uma grade de incerteza espacial do DCN em relação ao ITRF sem utilizar técnicas de interpolação?

Desafios quanto aos dados geodésicos e gravimétricos:

a) No projeto NEVREF, foram levantados os dados necessários para que se tenha um modelo geoidal com uma acurácia de $5 \mathrm{~mm}$ em terra (FARHANI, 2017). Qual deve ser a densificação dos dados gravimétricos para que no Brasil o referencial vertical terrestre, ao invés do NMM, seja uma superfície equipotencial com tal acurácia?

b) As estações maregráficas estão sujeitas aos movimentos verticais da crosta terrestre. Dessa forma, pergunta-se: as estações GNSS da RBMC seriam suficientes para modelar tais movimentos com uma acurácia sub-centimétrica? Havendo uma resposta negativa, quais seriam os locais recomendados para instalação de novas estações de monitoramento contínuo?

c) Quais recursos financeiros e humanos são necessários para que o posicionamento em tempo real no Brasil forneça uma acurácia centimétrica para plataformas flutuantes, via Network-RTK ou SBAS (Sattelite Based Augmentation System)?

d) Quais áreas seriam prioritárias para levantamentos gravimétricos e geodésicos?

e) Qual seria o retorno socioeconômico destes investimentos em dez anos? Para detalhes dos benefícios pode-se consultar NGS (2001) e NGS (2013).

\section{CONSIDERAÇÕES FINAIS}

Cartas náuticas com SEP contribuem para a Década dos Oceanos para que eles sejam seguros, previsíveis, transparentes e produtivos, de forma que os dados do nível do mar permitam uma batimetria e 
topografia integrada; redução das incertezas dos levantamentos hidrográficos; e navegação com uma superfície contínua do DCN, facilitando a integração do banco de dados hidrográficos, tanto no espaço, quanto no tempo. Países de dimensões continentais como Estados Unidos e Canadá iniciaram localmente até atingirem uma cobertura nacional e o resultado foi o cálculo da superfície do DCN em relação ao ITRF com incertezas próximas de $10 \mathrm{~cm}$. Já no Brasil, para a definição do DCN, utiliza-se a técnica do zoneamento de marés, que prevê uma incerteza de até $10 \mathrm{~cm}$ entre zonas adjacentes, estando referido ao NMML e adequado somente para pequenas áreas. Entretanto, em 2010, foram realizadas iniciativas para o cálculo do SEP na Baía de Guanabara, que podem servir de subsídios para outros levantamentos. A partir do Alt-Bat se tem uma perspectiva da cobertura nacional que, por sua vez, nasceu com o objetivo de integrar os referenciais terrestre e marítimo, mas sua aplicação se estende à hidrografia e à segurança da navegação.

Na utilização do geoide como superfície de referência vertical para os modelos hidrodinâmicos, é necessária uma maior acurácia do modelo geoidal ou quase-geoidal adotado no país. Neste sentido, o Navio $\mathrm{NPqO}$ Vital de Oliveira pode ser uma alternativa para a densificação de dados gravimétricos na região costeira, sendo necessário também o investimento em gravimetria aérea, terrestre e satelital, como um projeto de longo prazo.

Para a definição das superfícies médias do mar, MSS, foram citados o modelo global de marés canadense WebTideModel (seção 3.1.2), ou o modelo global oceânico CLS da Aviso+ (seção 3.1.2), ou DTU15MSS do DTU Space (seção 2.3). Estes dados sofrem deterioração nas proximidades da costa, mas podem ser ajustados por modelos hidrodinâmicos existentes.

Quanto ao DCN, sugere-se dividir o Brasil em regiões hidrográficas específicas e instalar uma rede de estações maregráficas com posições geocêntricas conhecidas em áreas onde a segurança navegação e o gerenciamento costeiro é crítico. Também é importante a definição de uma Época Nacional de Observação do Datum de Maré durante 18.6 anos. Além da realização de nivelamento geométrico das estações maregráficas durante esse período e bem como, a atualização dos equipamentos maregráficos, conforme preconizado em IBGE (2017). Ainda se recomenda a utilização da interpolação estatística dos Data de maré nos modelos hidrodinâmicos. Por fim, uma meta interessante a ser adotada seria o cálculo da incerteza espacial do DCN em relação ao ITRF, cujas incertezas deveriam ser melhores que $10 \mathrm{~cm}$ nas proximidades da costa.

\section{Agradecimentos}

Os autores agradecem à Marinha do Brasil por conceder ao autor principal a possibilidade de realizar o mestrado na Universidade Federal do Paraná (UFPR), por meio do acordo de cooperação número 87/2014 com o Centro de Hidrografia da Marinha.

À UFPR e ao Programa de Pós-Graduação em Ciências Geodésicas da Universidade Federal do Paraná (UFPR), em especial à professora Regiane Dalazoana pelas aulas de Geodésia básica ministradas.

Aos conselhos e referências enviados pessoalmente por e-mail: Catherine Robin and Shannon Nudds (Natural Resources Canada); Jonathan Illife (University College London); Cornellis Slobbe (Delft University of Technology); David Dodd (IIC Technologies); e Mohammed El-Diasty (King Abdulaziz University)

Às revisões realizadas: Nikko Yumul (Ateneo de Manila University); Marcelo Benites (PPGCL/UENF); e Jorge Euriques (UFPR).

Além dos dois revisores anônimos que muito contribuíram para o aprimoramento do trabalho.

\section{Contribuição dos Autores}

Felipe Rodrigues Santana realizou a conceptualização, redação da minuta inicial, redação da versão final, revisão e edição. Tulio Alves Santana: conceptualização, redação da minuta inicial, avaliação, revisão, edição e referências bibliográficas. Cláudia Pereira Kreuger: conceptualização, supervisão, avaliação, revisão e edição. Guilherme Antonio Gomes do Nascimento: avaliação, revisão e edição. Aluizio Oliveira Jr: avaliação, revisão e edição.

\section{Conflitos de Interesse}

Os autores declaram que não há conflitos de interesse. 


\section{Referências}

ABDULlAH, M. A. B.; OMAR, A, H. Geoid Based Seamless Vertical Height Datum for Marine Cadastre Application. Geoinformation Science Journal, Malasya, 2011. Disponível em:< https://mafiadoc.com/queue/geoid-based-seamless-vertical-height-datum-for_59e44c791723ddbdd515b761.html >. Acesso em: 24 jul. 2020.

ALTAMIMI, Z.; REBISCHUNG, P.; MÉTIVIER, L.; COLLILIEUX, X. Analysis and results of ITRF2014. 2017 (IERS Technical Note; 38) Frankfurt am Main: Verlag des Bundesamts für Kartographie und Geodäsie, 76 pp., ISBN 978-3-86482-088-5 (print version), 2017.

ADAMS, R. The Development of a Vertical Reference Surface and Model for Hydrography - a Guide. In: XXIII INTERNATIONAL FIG CONFERENCE, 2006, Munich, Germany. Proceedings... Munich, Germany: UK Hydrographic Office, 2006. p. 1-13.

ALVAREZ MACHUCA, M. C. Á.; PUlido NOSSA, D. A. P.; SOlANO TRULlO, L. J. S.; BARRERO OVIEDO, F. Construcción de la superficie hidrográfica de referencia vertical para las bahías de Buenaventura y Málaga, Pacífico colombiano. Boletín Científico Cioh, [s.1.], n. 36, p. 53-69, 12 dez. 2018. DOI.: $10.26640 / 22159045.438$.

ARENTZ, M. F. R. A Modelagem Hidrodinâmica Como Auxílio à Navegação no Canal Norte do Estuário do Amazonas. 2009. 166 f. Dissertação (Mestrado em Engenharia Oceânica) - COPPE, Universidade Federal do Rio de Janeiro, Rio de Janeiro, 2009.

ARGONÁUTICA. Redraft: Sistema de Calado Dinâmico. 2020. Disponível em:< https://argonautica.com.br/redraft/>. Acesso em: 25 jul. 2020.

BOSCH, W. Analytical Report for Integrating Hydrodynamic and Elevation Models. In: DIALOGOS SETORIAIS: UNIÃO EUROPEIA BRASIL. Rio de Janeiro, jun. 2016a. Disponível em:< https://www.concar.gov.br/temp/249@Analytical-Report_2016-07-18.pdf>. Acesso em: 25 jul. 2020.

BOSCH, W. European Experiences on Refinement, Connection, and Unification of Vertical Reference Surfaces in Coastal Zones. In: DIALOGOS SETORIAIS: UNIÃO EUROPEIA BRASIL. Rio de Janeiro, jun. 2016c. Disponível em:< https://www.concar.gov.br/temp/248@Report01final_Relat\%c3\%b3rio\%20preliminar.pdf>. Acesso em: 25 jul. 2019.

BOSCH, W. Evaluating Technical Issues for Reconciling Continental Altimetry and Ocean Bathymetry. In: DIALOGOS SETORIAIS: UNIÃO EUROPEIA BRASIL. Rio de Janeiro, jun. 2016b. Disponível em:< https://www.concar.gov.br/temp/250@CLIM0009-EvaluationReport.pdf>. Acesso em: 25 jul. 2020.

CANADIAN HYDROGRAPHIC SERVICE (CHS). Standards for Hydrographic Surveys. Survey Management Guidelines. 2013. Disponível em:< https://ppa.gc.ca/sites/default/files/201809/CHS\%20Standards\%20for\%20Hydrographic\%20Surveys.pdf>. Acesso em: 13 jul. 2020.

COLIN, J. Coastal Mapping Portal Demonstration. 2020. Disponível em:< http://www.searica.eu/documents/category/26-2017-02-07?download=173:170207-joseph-colinworldline.>. Acesso em: 30 jul. 2020.

COMISSÃO NACIONAL DE CARTOGRAFIA (CONCAR). Comitê de Integração dos Componentes $\begin{array}{llllll}\text { Verticais } & \text { Terrestre } & \text { e } & \text { Marítima. } & 2016 . & \text { Disponível }\end{array}$ em:〈https://www.concar.gov.br/detalheEntidades.aspx?cod=25>. Acesso em: 24 jul. 2020.

COMPANHIA DE PESQUISA DE RECURSOS MINERAIS (CPRM). CPRM debate utilização de novas tecnologias nos projetos de Geologia Marinha. 2019. Disponível em:< https://www.cprm.gov.br/publique/Noticias/CPRM-debate-utilizacao-de-novas-tecnologias-nos-projetosde-Geologia-Marinha-5806.html>. Acesso em: 24 jul. 2020.

CONSELHO NACIONAL DE PRATICAGEM (CONAPRA). Novo DPC, Almirante Roberto visita Praticagem do RJ e conhece o ReDRAFT. 2020. Disponível em:< https://www.praticagemdobrasil.org.br/novo-dpc-almirante-roberto-visita-praticagem-do-rj-e-conhece-o- 
redraft/>. Acesso em: 24 jul. 2020.

CENTER FOR OPERATIONAL OCEANOGRAPHIC PRODUCTS AND SERVICES (CO-OPS). Tidal Datums and their applications. NOAA Special Publication NOS CO-OPS 1. NOAA: Silver Spring, MD, USA, 2001. Disponível em <https://tidesandcurrents.noaa.gov/publications/tidal_datums_and_their_applications.pdf >. Acesso em: 13 nov. 2020.

INSTITUTO ALBERTO LUIZ COIMBRA DE PÓS-GRAUDAÇÃO E PESQUISA EM ENHENHARIA (COPPE). Pesquisadores da Coppe apresentam Big Data das Baías do Brasil. 2018. Disponível em https://coppe.ufrj.br/pt-br/planeta-coppe-noticias/noticias/pesquisadores-da-coppe-apresentam-big-datadas-baias-do-brasilAcesso em: 03 nov. 2020

DANMARKS TEKNISKE UNIVERSITET (DTU). DTU Space. 2020. Disponível em: < https://www.space.dtu.dk/english/research/scientific_data_and_models >. Acesso em: 20 jul. 2020.

DE LANGE BOOM, B. Vertical Datum Separation Models for the British Columbia Coast. In: CHC 2012 CONFERENCE. Niagara Falls, Canada. 2012.

DIRETORIA DE HIDROGRAFIA E NAVEGAÇÃO (DHN). Normas da autoridade marítima para levantamentos hidrográficos. $2^{\circ}$ Revisão. 2017. Disponível em: < https://www.marinha.mil.br/dhn/?q=pt-br/node/266 >. Acesso em: 13 jul. 2020.

DIRETORIA DE PORTOS E COSTAS (DPC). Normas da autoridade marítima para implantação e operação de sistemas para determinação de folga dinâmica abaixo da quilha. NORMAM-33/DPC. 2019. Disponível em: < https://www.marinha.mil.br/dpc/sites/www.marinha.mil.br.dpc/files/NORMAM33_DPC_0.pdf >. Acesso em: 20 jul. 2020.

DODD e MILSS, 2012. Ellipsoidally Referenced Surveys Separation Models. In: FIG WORKING WEEK 2012 Rome, Italy, 6-10 May 2012.

EL-DIASTY, M.; AL-HARBI, S.; PAGIATAKIS, S. Development of Saudi continuous chart datum: arabian gulf case study. Geomatics, Natural Hazards And Risk, [s.l.], v. 10, n. 1, p. 1738-1749, 1 jan. 2019. DOI.: $10.1080 / 19475705.2019 .1614682$.

ELLMER, W.; GOFFINET, P. Tidal Correction Using GPS - Determination of the Chart Datum. In: XXIII FIG CONGRESS, 2006. Munich. Proceedings..., Munich: 2006, p. 1-14.

FARAHANI, H. H., Klees, R., \& Slobbe, C. (2017). Data requirements for a 5-mm quasi-geoid in the Netherlands. Studia Geophysica et Geodaetica, 61(4), p.675-702. DOI.:10.1007/s11200-016-0171-7

FÉDÉRATION INTERNATIONALE DES GÉOMÈTRES (FIG). Ellipsoidally Referenced Surveying for Hydrography. N. 62. Copenhagen, Denmark. 2014. Disponível em: < https://www.fig.net/resources/publications/figpub/pub62/Figpub62.pdf >. Acesso em: 20 jul. 2020.

FÉDÉRATION INTERNATIONALE DES GÉOMÈTRES (FIG). FIG Guide on the Development of a Vertical Reference Surface for Hydrography. N. 37. Copenhagen, Denmark. 2006. Disponível em: <https://www.fig.net/resources/publications/figpub/pub37/pub37.pdf>. Acesso em: 13 jul. 2020.

FENG, G.; JIN, S.; ZHANG, T. Coastal sea level changes in Europe from GPS, tide gauge, satellite altimetry and GRACE, 1993-2011. Advances In Space Research, [s.1.], v. 51, n. 6, p. 1019-1028, mar. 2013. DOI. 10.1016/j.asr.2012.09.011

FINALISING SURVEYS FOR THE BALTIC MOTORWAYS OF THE SEA (FAMOS). Projeto FAMOS. 2020. Disponível em:< http://www.famosproject.eu/>. Acesso em: 15 jul. 2020.

FLECHTNER, F.; SCHUH, W.-D.; SNEEUW, N. Observation of the System Earth from Space - CHAMP, GRACE, GOCE and future missions. Berlin: Springer, 2014. 190 p. (GEOTECHNOLOGIEN Science Report No. 20).

FRANZ, G.; LUERSEN, D.; BONATTO, B.; PALOSCHI, N.; ITALINI, D.; RODRIGO, P.; E NOERNBERG, M. Sistema Operacional de Modelagem Hidrodinâmica para a Plataforma Sudeste do Brasil Observatório do Mar. In: XIII SIMPÓSIO SOBRE ONDAS, MARÉS, ENGENHARIA OCEÂNICA E OCEANOGRAFIA POR SATÉLITE. Arraial do Cabo, Brazil. 2018. 
GEORGAS, N.; WEN, B.; ZHAO, Y. Calculation of vertical tidal datums for the tidal Hudson River north of Yonkers, New York. Relatório Técnico Final. Stevens Institute of Technology. Disponível em: < https://web.stevens.edu/ses/documents/fileadmin/documents/pdf/Final_Report_20131220.pdf>. Acesso em: 30 jul. 2020.

GONZÁLEZ ACUÑA, J.; ARROYO SUAREZ, E. N. Comparative methodologies for sounding reduction applied to a bathymetric survey referenced to the WGS84 ellipsoid, executed in Concepcion Bay and Gulf of Arauco, VIII region, Chile. 2013. Disponível em:< https://www.yumpu.com/en/document/view/15374601/comparative-methodologies-for-soundingreduction-applied-to-a->. Acesso em: 24 jul. 2020.

GOVERNO DO ESTADO DO RIO GRANDE DO SUL. Plano de Desenvolvimento e zoneamento. Porto Alegre, 2019. Disponível em:

<http://antigo.infraestrutura.gov.br/images/SNP/planejamento_portuario/pdz/pdz24.pdf>. Acesso em: 01 nov. 2020.

GRGIć, M.; NEREM, R. S.; BAŁIć, T. Absolute Sea Level Surface Modeling for the Mediterranean from Satellite Altimeter and Tide Gauge Measurements. Marine Geodesy, [s.1.], v. 40, n. 4, p. 239-258, 22 jun. 2017. DOI.: 10.1080/01490419.2017.1342726.

HAINS, D. Success Depends on the Quantity and Quality of All Available Data. Preparing for Hydrographic Artificial Intelligence Network and Systems. Hydro international. p. 16-17, july/august, 2019.

HESS, K. W. Spatial Interpolation of Tidal Data in Irregularly-shaped Coastal Regions by Numerical Solution of Laplace's Equation. Estuarine, Coastal And Shelf Science, [s.1.], v. 54, n. 2, p. 175-192, fev. 2002. DOI.: 10.1006/ecss.2001.0838.

HUGLES, C. W.; BINGHAM, R. J. An Oceanographer's Guide to GOCE and the Geoid. Ocean Science, [s.1.], v. 4, n. 15, p.15-29, 2008.

ILIFFE, J. C.; ZIEBART, M. K.; TURNER, J. F.. A New Methodology for Incorporating Tide Gauge Data in Sea Surface Topography Models. Marine Geodesy, [s.1.], v. 30, n. 4, p.271-296, 7 nov. 2007. DOI. 10.1080/01490410701568384.

ILIFFE, J. C.; ZIEBART, M. K; TURNER, J. F.; TALBOT, A. J.; LESSNOFF, A. P. Accuracy of vertical datum surfaces in coastal and offshore zones. Survey Review, [s. 1.], v. 45, n. 331, p. 254-262, jul. 2013. DOI.: 10.1179/1752270613y.0000000040.

INSTITUTO BRASILEIRO DE GEOGRAFIA E ESTATÍSTICA (IBGE). Especificações e Normas para Levantamentos Geodésicos associados ao Sistema Geodésico Brasileiro. Rio de Janeiro. 2017. Disponível

<ftp://geoftp.ibge.gov.br/metodos_e_outros_documentos_de_referencia/normas/normas_levantamentos_ geodesicos.pdf >. Acesso em: 27 dez. 2019

INSTITUTO BRASILEIRO DE GEOGRAFIA E ESTATÍSTICA (IBGE). Reajustamento da Rede Altimétrica com Números Geopotenciais 2018. Rio de Janeiro: IBGE, 2018. Disponível em: <ftp://geoftp.ibge.gov.br/informacoes_sobre_posicionamento_geodesico/rede_altimetrica/relatorio/relato rio_REALT_2018.pdf>. Acesso em: 24 jul. 2020.

INTERGOVERNMENTAL OCEANOGRAPHIC COMMISSION OF UNESCO (IOC/UNESCO); INTERNATIONAL MARITIME ORGANISATION (IMO); FOOD AND AGRICULTURE ORGANIZATION OF UNITED NATIONS (FAO); UNITED NATIONS DEVELOPMENT PROGRAMME (UNDP). A Blueprint for Ocean and Coastal Sustainability. Paris. 2011. Disponível em:

https://www.undp.org/content/dam/undp/library/Environment\%20and\%20Energy/Water\%20and\%20Oc ean\%20Governance/interagency_blue_paper_ocean_rioPlus20.pdf >. Acesso em: 20 jul. 2020.

INTERNATIONAL ASSOCIATION OF GEODESY (IAG). Resolution (No. 1) for the definition and realization of an International Height Reference System (IHRS). München, Alemanha, 2015. Disponível em: <https://iag.dgfi.tum.de/fileadmin/IAG-docs/IAG_Resolutions_2015.pdf>. Acesso em: 13 jul. 2020. 
KE, H.; LI, F.; AI, S.; LEI, J.; WANG, Z.; ZHANG, S. Establishment of Chart Datum and Vertical Datum Transformation for Hydrography in the Chinese Great Wall Bay, Antarctic Peninsula. Journal Of Surveying Engineering, [s.1.], v. 146, n. 2, p. 1-12, maio 2020. DOI.: 10.1061/(asce)su.19435428.0000312.

KEYSERS, J.H.; QUADROS, N.D.; COLLIER, P.A. Vertical datum transformations across the Australian littoral zone. Journal of Coastal Research, Coconut Creek, v. 31, n. 1, p. 119-128, 2015.

KRUEGER, C. P. Investigações sobre aplicações de alta precisão do GPS no âmbito marinho. 1996. 288 f. Tese (Doutorado em Ciências Geodésicas) - Setor de Ciências da Terra, Universidade Federal do Paraná, Curitiba, 1996.

LEE, W.; CHOI, Y.; HAN, K.; PARK, H. Construction of Tidal Datums Based on Ellipsoid Using Spatial Interpolation. In: FIG WORKING WEEK 2017. Helsinki, Finland. 2017.

LEFAIVRE, D.; GODIN, A.; DODD, D.; HERRON, T. The Continuous Vertical Datum Canadian Waters Project (Beginnings, Vision, Methods and Progress). In: CHC 2010 CONFERENCE. Quebec, QC, Canada. 2010.

LI, S.; FUNG, K.S. Maritime autonomous surface ships (MASS): implementation and legal issues. Maritime Business Review, [S.1.], v. 4, n. 4, p. 330-339, 18 nov. 2019. DOI.: 10.1108/mabr-01-2019-0006.

LUZ, R. T. Cálculo de altitudes científicas e sua aplicação no reajustamento da Rede Altimétrica de Alta Precisão do Sistema Geodésico Brasileiro. Revista Brasileira de Geografia, Rio de Janeiro, v. 61, n. 1, p.79-97, jun. 2016.

LUZ, R. Vinculação entre os níveis de referência altimétricos e batimétricos para o posicionamento vertical onshore e offshore e o estudo dos impactos da elevação do NMM na zona costeira. In: VII SIMGEO. Recife, Brasil. 09 de Novembro de 2018.

MARINHA DO BRASIL. Centro de Hidrografia da Marinha. Sondagem Batimétrica. 2020. Disponível em: < https://www.marinha.mil.br/chm/dados-do-segnav-lev-hidro/sondagem-batimetrica>. Acesso em: 24 jul. 2020.

MARTIN, R. J.; BROADBENT, G. J. Chart Datum for Hydrography. The Hydrographic Journal, [s.l.], n. 112, p. 9-14, abril. 2004.

MATOS, A. C. O. C.; BLITZKOW, D.; GUIMARÃES, G. DO N.; LOBIANCO, M. C. B.; COSTA, S. M. A. Validação do MAPGEO2010 e comparação com modelos do geopotencial recentes. Boletim de Ciências Geodésicas, Curitiba, v. 18, n. 1, p. 101-122, 2012. DOI.: 10.1590/s1982-21702012000100006.

MINISTÉRIO DO MEIO AMBIENTE (MMA). Programa Nacional para Conservação da Linha de Costa - PROCOSTA. Brasília: MMA, 2018. Disponível em: < https://www.mma.gov.br/publicacoes/gestaoterritorial/category/197-gest\%C3\%A3o-costeira-procosta.html?download=1443:programa-nacionalpara-conserva\%C3\%A7\%C3\%A3o-da-linha-de-costa >. Acesso em: 24 jul. 2020.

MYERS, E. VDatum Development, Upgrades, and Future. In: ADCIRC USERS GROUP MEETING 2018. [s.1.]. Maio, 2018. Disponível em: < https://cdr.lib.unc.edu/concern/scholarly_works/x920g2067 >. Acesso em: 29 jul. 2020.

MYERS, E.; HESS, K.; YANG, Z.; XU, J.; WONG, A.; DOYLE, D.; WOOLARD, J.; WHITE, S.; LE, B.; GILL, S.; HOVIS, G. VDatum and strategies for national coverage. In: MARINE TECHNOLOGY SOCIETY/IEEE OCEANS CONFERENCE, 2007. Vancouver, British Columbia, Canada. Proceedings..., Vancouver, British Columbia, Canada, 2007, p. 1-8.

MYERS, E.P.; WONG, A.; HESS, K.; WHITE, S.; SPARGO, E.; FEYEN, J.; YANG, Z.; RICHARDSON, P.; AUER, C.; SELLARS, J. Development of a national VDatum, and its application to sea level rise in North Carolina. In: THE U. S. HYDROGRAPHIC CONFERENCE, 2005. San Diego, CA, USA. Proceedings..., San Diego, CA, USA, 2005, p. 29-31.

NASCIMENTO, G. A. G. Verificação da Aplicabilidade de Dados Obtidos por Sistema Laser Batimétrico Aerotransportado à Cartografia Náutica. 2019. 112 f. Dissertação (Mestrado em Ciências Cartográficas) - Faculdade de Ciências e Tecnologia, Universidade Estadual "Júlio de Mesquita Filho", 
Presidente Prudente, 2019.

NATIONAL OCEANIC AND ATMOSPHERIC ADMINISTRATION (NOAA). Documentation for VDatum (and the Datum Tutorial). Vertical Datum Transformation Software Version 1.06. 2004. Disponível em: <https://vdatum.noaa.gov/download/publications/2002_milbert_VDatum106.pdf>. Acesso em: 20 jul. 2020.

NATIONAL OCEANIC AND ATMOSPHERIC ADMINISTRATION (NOAA). Mapping and Charting. 2020a. Disponível em:< https://tidesandcurrents.noaa.gov/mapping.html >. Acesso em: 25 fev. 2020.

NATIONAL OCEANIC AND ATMOSPHERIC ADMINISTRATION (NOAA). VDATUM. $2020 \mathrm{~b}$. Disponível em:< https://vdatum.noaa.gov/ >. Acesso em: 15 jul. 2020.

NATIONAL OCEANIC AND ATMOSPHERIC ADMINISTRATION (NOAA). VDATUM: Webinar Séries. 2018. Disponível em:< https://geodesy.noaa.gov/web/science_edu/webinar_series/vdatum_01-1118.shtml>. Acesso em: 29 jul. 2020.

NUDDS, S.; ROBIN, C.; MACAULAY, P. Continuous vertical datum separations for Canadian Waters: Creating Canada's first hydrographic vertical separation surfaces. Sea Technology, v. 57, n. 9, p. 38-42, 2016.

NATIONAL GEODETIC SURVEY (NGS). Socio-Economic Benefits Study: Scoping the Value of CORS and GRAV-D. 2009. Disponível em <:https://www.ngs.noaa.gov/PUBS_LIB/SocioEconomicBenefitsofCORSandGRAV-D>. Acesso em: 13 nov. 2020.

NATIONAL GEODETIC SURVEY (NGS). Ten years strategic plan 2013 - 2023. Positioning America For the Future. 2013. Disponível em <https://www.ngs.noaa.gov/web/news/Ten_Year_Plan_2013-2023.pdf. >. Acesso em: 13 nov. 2020.

O'REILLY, C.; PARSONS, S.; LANGELIER. A Seamless Vertical Reference Surface for Hydrographic Data Acquisition and Information Management. In: CANADIAN HYDROGRAPHIC CONFERENCE. Jun. 1996. p. 26-33.

ODAMAKI, M.; NAKAMURA, H. Proposal on Integrated Database of Sounding Depth, Height and Mean Sea Level on Reference Ellipsoid, and A Trial of Time Variable Depth Display on ECDIS. Relatório Técnico. Disponível em: < https://www.oceandocs.org/handle/1834/16587>. Acesso em: 20 jul. 2020.

OLIVEIRA JUNIOR, A. M.; ARROYO, E. N. S.; RAMOS, A. M.; ARENTZ, M. F. R. Seabed Mapping on an Earth Centered Earth Fixed (ECEF) Geocentric Reference Frame. Cooperative Validation with US Navy and Brazilian Navy in Guanabara Bay, Rio de Janeiro. In: ION GNSS 2010 CONFERENCE. Portland OR USA. September 21 - 24, 2010.

OREIRO, F.; D’ONOFRIO, E.; FIORE, M. Altimetric Reference Connection of Nautical Charts And WGS84 Ellipsoid For The Río de La Plata. Geoacta, Buenos Aires, v. 40, n. 2, p. 109--120, 2016.

ORGANIZAÇÃO HIDROGRÁFICA INTERNACIONAL (OHI). IHO Guidelines for Creating S-100 Product Specifications. Principauté de Monaco. 2020b. Disponível em: $<$ https://iho.int/en/s-100-basedproduct-specifications >. Acesso em: 20 jul. 2020.

ORGANIZAÇÃO HIDROGRÁFICA INTERNACIONAL (OHI). IHO Standards for Hydrographic Surveys. Principauté de Monaco. 2020a. Disponível em: $<$ https://iho.int/uploads/user/pubs/Drafts/S44_Edition_6.0.0-Final.pdf >. Acesso em: 29 jul. 2020.

ORGANIZAÇÃO HIDROGRÁFICA INTERNACIONAL (OHI). S-32 IHO Hydrographic Dictionary. Principauté de Monaco. 2020c. Disponível em: $<$ http://ihoohi.net/S32/engView.php?quick_filter=tide+heights\&quick_filter_operator=Contains>. Acesso em: 06 ago. 2020.

OSILIERI, P. R. G.; SEOANE, J. C. S.; DIAS, F. F. Coastal Vulnerability Index revisited: a case study from maricá, rj, brazil. Revista Brasileira de Cartografia, Uberlândia, v. 72, n. 1, p. 81-99, 30 mar. 2020. DOI.: $10.14393 /$ rbcv72n1-47025.

PARKER, B. The Integration of Bathymetry, Topography and Shoreline and the Vertical Datum 
Transformations behind It. International Hydrographic Review, v. 3, n. 3. p. 14-26. 2002.

PARKER, B.; MILBERT, D.; HESS, K.; GILL, S. National Vdatum - The Implementation of a National Vertical Datum Transformation Database. National Ocean Service. 2003. Disponível em:< https://vdatum.noaa.gov/download/publications/2003_parker_USHydro_nationalvdatum.pdf $>$. Acesso em: 20 jul. 2020.

PINEAU-GUILlOU, L. Set-up of CD using spatial altimetry and GPS. 2009. Disponível em:< https://legacy.iho.int/mtg_docs/com_wg/IHOTC/TWLWG\%201/France.pdf >. Acesso em: 24 jul. 2020.

PINEAU-GUILLOU, L.; DORST, L. Creation of Vertical Reference Surfaces at Sea Using Altimetry and GPS. In: ALTAMIMI, Z.; COLLILIEUX, X. Reference Frames for Applications in Geosciences. v. 138, 2012. p.229-235. DOI. 10.1007/978-3-642-32998-2.

PLAG, H-P.; BEUTHER, G.; GROSS, R.; HERRING, T. A.; RIZOS, C.; RUMMER, R.; SAHAGIAN, D.; ZUMBERGE, J. Introduction. In: Plag, H-P. Pearlman, M. The Global Geodetic Observing System: Meeting the Requirements of a Global Society on a Changing Planet in 2020. New York: Springer, 2009. p. 1-13. DOI: 10.1007/978-3-642-02687-4.

RAMOS, A. M. Aplicação, Investigação e Análise da Metodologia de Reduções Batimétricas Através do Método GPS Diferencial Preciso. 2007. 227 f. Dissertação (Mestrado em Ciências Geodésicas) - Setor de Ciências da Terra, Universidade Federal do Paraná, Curitiba, 2007.

REIS, V. P.; BARBOSA, L. G.; PALMEIRO, A. S. Determinação da topografia do nível médio do mar com altimetria por satélites. Revista Brasileira de Geomática, Curitiba, v. 6, n. 2, p. 77, 21 maio 2018. DOI.: 10.3895/rbgeo.v6n2.5517.

RICE, G.; RILEY, J. Measuring the Water Level Datum Relative to the Ellipsoid During Hydrographic Survey. In: U. S. HYDROGRAPHIC CONFERENCE, 2011. Proceedings... Tampa, Florida, 2011, p. 1-11.

ROBIN, C.; GODIN, A.; MACAULAY, P.; DE LANGE BOOM, B.; LEFAIVRE, D.; HERRON, T.; SINNOTT, D.; BALLANTYNE, A.; MALTAIS, L.; VÉRONNEAU, M. The Canadian Hydrographic Continuous Vertical Datum: Methodology and Accuracy. In: CHC 2012 CONFERENCE. Niagara Falls, Canada. 2012.

ROBIN, C.; MACAULAY, P.; NUDDS, S.; GODIN, A.; DE LANGE BOOM, B.; BARTLETT, J.; MALTAIS, L.; HERRON, T.; CRAYMER, M.; VÉRONNEAU, M.; FADAIE, K. Modeling tidal water levels for Canadian coastal and offshore waters: implications for coastal change and adaptation. In: AGU FALL MEETING SAN FRANCISCO, December 15-19, 2014. Paper NH53A-3876.

ROBIN, C.; NUDDS, S.; MACAULAY, P.; GODIN, A.; DE LANGE BOOM, B; BARTLETT, J. Hydrographic Vertical Separation Surfaces (HyVSEPs) for the Tidal Waters of Canada. Marine Geodesy, [s.1.], v. 39, n. 2, p. 195-222, 3 mar. 2016. DOI.: 10.1080/01490419.2016.1160011.

SÁNCHEZ, L.; CUNDERLÍK, R.; DAYOUB, N.; MIKULA, K.; MINARECHOVÁ, Z.; SÍMA, Z.; VATRT, V. VOJTÍSKOVÁ, M. A conventional value for the geoid reference potential $W_{0}$. Journal of Geodesy, [s.1.], v. 90, n. 9, p.815-835, 23 maio 2016. DOI.10.1007/s00190-016-0913-x.

SÁNCHEZ, L.; SIDERIS, M. G. Vertical datum unification for the International Height Reference System (IHRS). Geophysical Journal International, [s.1.], p.570-586, 21 jan. 2017. DOI. 10.1093/gji/ggx025.

SANTANA, T. A. Contribuições para os Estudos de Integração das Componentes Verticais Terrestre e Marinha ao Longo da Costa Brasileira. 2020. 140 f. Dissertação (Mestrado em Ciências Geodésicas) Setor de Ciências da Terra, Universidade Federal do Paraná, Curitiba, 2020.

SANTANA, T. A.; DALAZOANA, R.. Integração dos Referenciais Verticais Terrestre e Oceânico: conceitos relacionados, projetos desenvolvidos e desafios. Revista Brasileira de Cartografia, Uberlândia, v. 72, n. 2, p. 345-364, 22 jun. 2020. DOI.: 10.14393/rbcv72n2-52611.

SHI, L.; HESS, K. W.; MYERS, E. P. Spatial Interpolation of Tidal Data Using a Multiple-Order Harmonic Equation for Unstructured Grids. International Journal Of Geosciences, [s.1.], v. 04, n. 10, p. 1425-1437, 2013. DOI.: 10.4236/ijg.2013.410140.

SHI, L.; MYERS, E. Statistical Interpolation of Tidal Datums and Computation of Its Associated Spatially 
Varying Uncertainty. Journal Of Marine Science And Engineering, [s.1.], v. 4, n. 4, p. 64, 22 set. 2016. DOI.: $10.3390 /$ jmse4040064.

SILVA, D. Condições Metereológicas e Ambientais. In: $7^{\circ}$ ENCONTRO SOBRE NORMAS DA AUTORIDADE MARÍTIMA PARA LEVANTAMENTOS HIDROGRÁFICOS. Rio de Janeiro. 21 e 22 de Novembro de 2019.2 Disponível em: <https://www.marinha.mil.br/chm/sites/www.marinha.mil.br.chm/files/u2009/condicoes_meteorologicas _e_ambientais.pdf >. Acesso em: 25 jul. 2020.

SIMON, B. Coastal Tides. Prince de Monaco: Institut océanographique, 2013. 413 p.

SLOBBE, D. C. Roadmap to a mutually consistent set of offshore vertical reference frames. Netherlands: Ncg, Nederlandse Commissie Voor Geodesie, Netherlands Geodetic Commission, Delft, The Netherlands, 2013. 263 p. Disponível em: https://ncgeo.nl/downloads/82Slobbe.pdf. Acesso em: 06 dez. 2020.

SLOBBE, D. C.; KLEES, R. Establishing a consistent vertical reference frame for the North Sea area. Relatório. ago. 2012. Disponível em:< http://blast-project.eu/media.php?file=623 >. Acesso em: 20 jul. 2020.

SLOBBE, D. C.; KLEES, R. Establishing a consistent vertical reference frame for the North Sea area. Relatório. ago. 2012. Disponível em:< http://blast-project.eu/media.php?file=623 >. Acesso em: 25 jul. 2019.

SLOBBE, D. C.; KLEES, R.; GUNTER, B. C.. Realization of a consistent set of vertical reference surfaces in coastal areas. Journal Of Geodesy, [s.1.], v. 88, n. 6, p. 601-615, 26 mar. 2014. DOI.: 10.1007/s00190014-0709-9.

SLOBBE, D. C.; KLEES, R.; VERLAAN, M.; ZIJL, F.; ALBERTS, B.; FARAHANI, H. H.. Height system connection between island and mainland using a hydrodynamic model: a case study connecting the dutch wadden islands to the amsterdam ordnance datum (nap). Journal Of Geodesy, [s.1.], v. 92, n. 12, p. 14391456, 9 mar. 2018. DOI.: 10.1007/s00190-018-1133-3.

SLOBBE, D. C.; SUMIHAR, J.; FREDERIKSE, T.; VERLAAN, M.; KLEES, R.; ZIJL, F.; FARAHANI, H. H.; BROEKMAN, R. A Kalman Filter Approach to Realize the Lowest Astronomical Tide Surface. Marine Geodesy, v. 41, n. 1, p. 44-67. 2018. DOI. 10.1080/01490419.2017.1391900.

SLOBBE, D. C.; SUMIHAR, J.; FREDERIKSE, T.; VERLAAN, M.; KLEES, R.; ZIJL, F.; FARAHANI, H. H.; BROEKMAN, R. A Kalman Filter Approach to Realize the Lowest Astronomical Tide Surface. Marine Geodesy, [s.1.], v. 41, n. 1, p. 44-67, 12 out. 2017. DOI.: 10.1080/01490419.2017.1391900.

SLOBBE, D. C.; KLEES, R.; VERLAAN, M.; DORST, L. L.; GERRITSEN, H. Lowest Astronomical Tide in the North Sea Derived from a Vertically Referenced Shallow Water Model, and an Assessment of its Suggested Sense of Safety. Marine Geodesy, v. 36, n. 1, p.31-71, 2013. DOI.: 10.1080/01490419.2012.743493

SOARES, S.; SANTOS, E. G.; SIQUEIRA, J. C. S.; LIMA, M. K.; LUZ, R. T. Rede Geodésica de Referência Costeira: Ações preliminares para integração de altitudes e profundidades. In: XIII SIMPÓSIO SOBRE ONDAS, MARÉS, ENGENHARIA OCEÂNICA E OCEANOGRAFIA POR SATÉLITE, 2009. Anais... Instituto de Estudos do Mar Almirante Paulo Moreira: Arraial do Cabo, RJ, 2009, p. 1-5.

GOVERNO DO ESTADO DO RIO GRANDE DO SUL (GERGS). Plano de Desenvolvimento e zoneamento. Porto Alegre, 2019. Disponível em: < http://antigo.infraestrutura.gov.br/images/SNP/planejamento_portuario/pdz/pdz24.pdf >. Acesso em: 01 nov. 2020.

TURNER, J. F.; ILIFFE, J. C.; ZIEBART, M. K. WILSON, C.; HORSBURGH, J. Interpolation of Tidal Levels in the Coastal Zone for the Creation of a Hydrographic Datum. Journal of Atmospheric and Oceanic Technology, [s.1.], v. 27, n. 3, p.605-613, mar. 2010. DOI. 10.1175/2009jtecho645.1.

TURNER, J. F.; ILIFFE, J. C.; ZIEBART, M. K.; JONES, C. Global Ocean Tide Models: Assessment and Use within a Surface Model of Lowest Astronomical Tide. Marine Geodesy, [s.1.], v. 36, n. 2, p.123-137, jan. 2013. DOI. 10.1080/01490419.2013.771717. 
UNITED NATIONS EDUCATIONAL, SCIENTIFIC AND CULTURAL ORGANISATION (UNESCO). A ciência que precisamos para o oceano que queremos. Década das Nações Unidas da Ciência Oceânica para o Desenvolvimento Sustentável (2021-2030). 2019. Disponível em: < http://ciencianomar.mctic.gov.br/wp-content/uploads/2020/06/265198por.pdf>. Acesso em: 13 jul. 2020.

VALE. Navio de pesquisa patrocinado pela Vale é entregue no Rio de Janeiro. 2015. Disponível em: < http://www.vale.com/brasil/pt/aboutvale/news/paginas/navio-pesquisa-patrocinado-vale-entregue-riojaneiro.aspx>. Acesso em: 30 jul. 2020.

WELLS, D. E.; KLEUSBERG, A.; VANÍČEK, P. A Seamless Vertical-Reference Surface for Acquisition, Management and ECDIS Display of Hydrographic Data. Canadá: University of New Brunswick, 1996. 74p. Relatório técnico.

WIŚNIEWSKI1, B.; WOLSKI, T.; GIZA, A. Adjustment of the European Vertical Reference System for the representation of the Baltic Sea water surface topography. Scientific Journals Maritime University of Szczecin, [s.1.], v. 38, n. 110, p. 106-117, 2014.

ZIEBART, M.; ILIFFE, J.; TURNER, J.; OLIVEIRA, J.; ADAMS, R. VORF - The UK Vertical Offshore Reference Frame: Enabling Real-time Hydrographic Surveying. THE 20TH INTERNATIONAL TECHNICAL MEETING OF THE SATELLITE DIVISION OF THE INSTITUTE OF NAVIGATION (ION GNSS 2007). Proceedings... Fort Worth, TX, September 2007, pp. 1943-1949.

\section{Biografias dos autores}

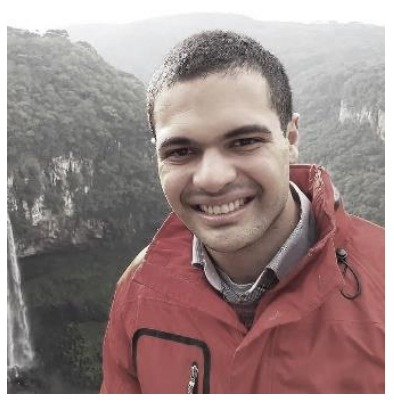

Felipe Rodrigues Santana, natural do Rio de Janeiro, RJ. É Capitão-Tenente da Marinha do Brasil, bacharel em Ciências Navais pela Escola Naval (2008-2011), e pós-graduação em Hidrografia pela Diretoria de Hidrografia e Navegação (DHN). Fez Curso para o desenvolvimento de habilidades internacionais e liderança social (2017 - Itália e 2018 - Suiça). Já realizou diversos levantamentos hidrográficos ao longo do brasileiro e em águas interiores como Baía de Guanabara, Baía de Sepetiba e Rio Guaíba. Atualmente é aluno de mestrado no curso de Ciências Geodésicas da Universidade Federal do Paraná (UFPR) (2019-2021).

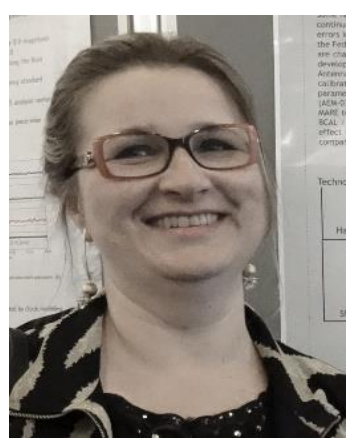

Claudia Pereira Krueger, nascida em Curitiba, Paraná, é engenheira civil pela UFPR (Universidade Federal do Paraná), Curitiba, Paraná., Brasil. Mestre em Ciências Geodésicas pela UFPR (1994); Doutora em Ciências Geodésicas, UFPR, com período de pesquisa desenvolvido junto ao Institut für Erdmesung, Universidade de Hannover (UH), Alemanha (1996) e Hidrógrafa Honorária pela Marinha do Brasil (2009). Atualmente, é docente do departamento de Geomática e dos programas de pós-graduação em Ciências Geodésicas (PPGCG) e em Engenharia de Recursos Hídricos e Ambiental (PPGERHA) da UFPR. Coordena o Laboratório de Geodésia Espacial e Hidrografia (LAGEH) e atua com pesquisas em Geodésia Espacial, Hidrografia e Posicionamento GNSS.

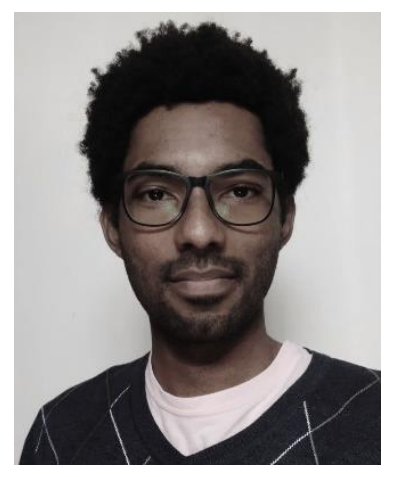

Tulio Alves Santana nasceu em Paracatu, no noroeste mineiro, em 1995. Cursou Engenharia de Agrimensura e Cartográfica na Universidade Federal de Uberlândia, campus Monte Carmelo (UFU/MC) (2013-2017). Fez mestrado acadêmico em Ciências Geodésicas na Universidade Federal do Paraná (UFPR) (2018-2020). Atualmente é Engenheiro Agrimensor e Cartógrafo na UFU/MC (Desde 2019) e cursa doutorado em Ciências Geodésicas da UFPR. Tem interesse nos seguintes temas: Integração dos referenciais verticais em zonas costeiras; Sistemas de monitoramento do planeta Terra; Referenciais Verticais; Integração de dados de Altimetria por satélite com dados maregráficos; Monitoramento do nível do mar; Modelagem do geopotencial; Conexão de data verticais; e Rede de Referência Altimétrica Internacional. 


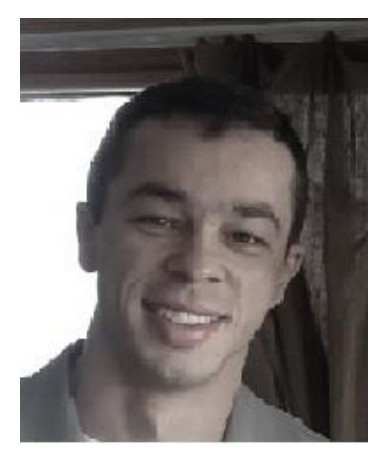

Guilherme A. G. do Nascimento é natural de Niterói-RJ. Capitão de Corveta da Marinha do Brasil, é bacharel em Ciências Navais pela Escola Naval, aperfeiçoado em Hidrografia pela Diretoria de Hidrografia e Navegação e mestre em Ciências Cartográficas pela Universidade Estadual Paulista. Realizou diversos levantamentos hidrográficos ao longo do litoral brasileiro e na região amazônica. Atualmente, é o encarregado da Seção de Informações Topogeodésicas do Centro de Hidrografia da Marinha, em Niterói-RJ.

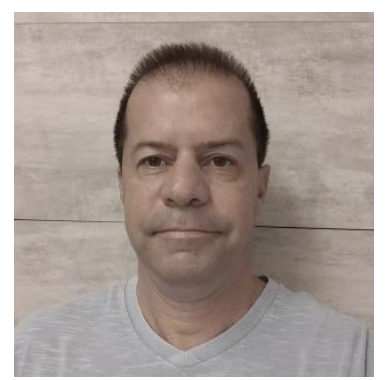

Aluizio Oliveira Jr., Capitão de Mar e Guerra da reserva da Marinha, também PhD em Geologia Marinha. Ele vem trabalhando em Hidrografia desde 1998, especialmente com ecobatímetros multifeixe, a bordo de navios e como encarregado da divisão de batimetria do Centro de Hidrografia da Marinha. Premiado com a medalha "Príncipe Albert I" da Organização Hidrográfica Internacional em 2012. Agora é o Diretor Técnico da empresa de sondagem Delfos.

Esta obra está licenciada com uma Licença Creative Commons Atribuição 4.0 Internacional - CC BY. Esta licença permite que outros distribuam, remixem, adaptem e criem a partir do seu trabalho, mesmo para fins comerciais, desde que lhe atribuam o devido crédito pela criação original. 